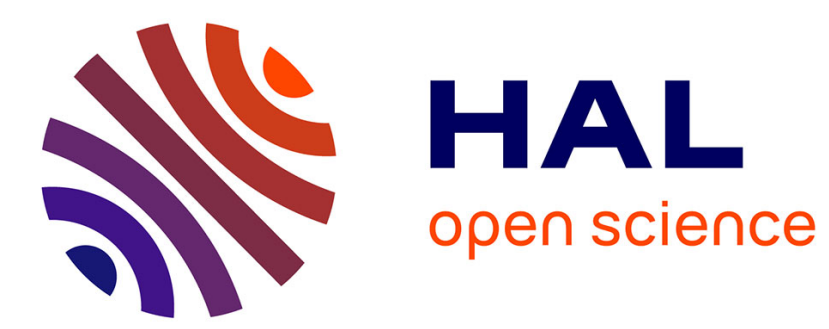

\title{
Quotients résolubles ou nilpotents des groupes de Kähler orbifoldes
}

\author{
Fédéric Campana
}

\section{To cite this version:}

Fédéric Campana. Quotients résolubles ou nilpotents des groupes de Kähler orbifoldes. 2009. hal00370560

\author{
HAL Id: hal-00370560 \\ https://hal.science/hal-00370560 \\ Preprint submitted on 24 Mar 2009
}

HAL is a multi-disciplinary open access archive for the deposit and dissemination of scientific research documents, whether they are published or not. The documents may come from teaching and research institutions in France or abroad, or from public or private research centers.
L'archive ouverte pluridisciplinaire HAL, est destinée au dépôt et à la diffusion de documents scientifiques de niveau recherche, publiés ou non, émanant des établissements d'enseignement et de recherche français ou étrangers, des laboratoires publics ou privés. 


\title{
QUOTIENTS RÉSOLUBLES OU NILPOTENTS DES GROUPES DE KÄHLER ORBIFOLDES
}

\author{
Frédéric Campana
}

26 février 2009

\section{Table des matières}

1 Introduction $\quad 2$

2 Rappels sur les orbifoldes géométriques et leurs revêtements. $\quad 3$

2.1 Orbifoldes géométriques . . . . . . . . . . . . . . . 4

2.2 Groupe fondamental . . . . . . . . . . . . . . . . . 5

2.3 Revêtement universel orbifolde . . . . . . . . . . . . . . 5

3 Ensembles de Green-Lazarsfeld des orbifoldes géométriques. $\quad 7$

3.1 Ensembles de Green-Lazarsfeld : généralités. . . . . . . . . . . . . . 7

3.2 Ensembles de Green-Lazarsfeld orbifoldes. . . . . . . . . . . . . . . . 7

4 Quotients résolubles des groupes de Kähler orbifoldes. 14

5 Morphisme d'Albanese. $\quad 17$

5.1 Factorisation de Stein du morphisme d'Albanese. . . . . . . . . . . . 17

5.2 Green-Lazarsfeld et morphisme d'Albanese. . . . . . . . . . . . . . . 17

5.3 Quotients résolubles et morphisme d'Albanese. . . . . . . . . . . . . 18

6 Orbifoldes spéciales. 21

$\begin{array}{lll}7 & \text { Réduction algébrique. } & 22\end{array}$

8 Quotients nilpotents des groupes de Kähler. 25

8.1 Rappels sur quotients et groupes nilpotents. . . . . . . . . . . . . . . 25

8.2 Morphismes stricts de groupes . . . . . . . . . . . . . . . 26

8.3 Fibrations et classe de nilpotence des groupes de Kähler. . . . . . . . 28

9 Bibliographie $\quad 29$ 


\section{Introduction}

- L'objectif du présent texte est d'étendre aux groupes fondamentaux des orbifoldes géométriques finies Kählériennes $(X \mid \Delta)$ les résultats connus concernant les quotients résolubles des groupes fondamentaux et les revêtements universels des variétés Kählériennes compactes. Cette extension se fait en adaptant au contexte orbifolde les arguments utilisés en l'absence de structure orbifolde.

- Les orbifoldes géométriques $(X \mid \Delta)$ (voir $\S 2.1$ ci-dessous) sont des revêtements ramifiés virtuels des variétés Kählériennes compactes $X$, et interviennent de manière essentielle dans leur classification biméromorphe (voir [Ca 07] et [Ca 04]) et, en particulier, dans l'étude de leurs groupes fondamentaux. Par exemple, si $f: Y \rightarrow$ $X$ est une fibration entre variétés Kählériennes compactes et connexes, de fibre générique $F$, on peut lui associer une orbifolde géométrique $\left(X \mid \Delta_{f}\right)$, appelée sa base orbifolde, déduite du diviseur des fibres multiples de $f$, telle que la suite suivante soit exacte ${ }^{1}$ :

$$
\pi_{1}(F) \rightarrow \pi_{1}(X) \rightarrow \pi_{1}\left(X \mid \Delta_{f}\right) \rightarrow 1
$$

Appliquée par exemple à la $\Gamma$-réduction (ou morphisme de Schafarevitch) de $X$, cette suite exacte réduit l'étude de $\pi_{1}(X)$ et de son revêtement universel à celle des mêmes objets pour les orbifoldes géométriques de type $\pi_{1}$-général (c'est-à-dire : dont le revêtement universel (voir §2.3) n'est pas recouvert par des sous-variétés compactes de dimension strictement positive).

- Les deux questions que nous souhaitons étudier sont ([Ca 07, question 12.12]) :

1. $\pi_{1}(X \mid \Delta)$ est-il commensurable à $\pi_{1}(Y)$, pour $Y$ compacte Kähler lisse adéquate?

2. Le revêtement universel $(\widetilde{X \mid \Delta})$ est-il isomorphe au revêtement universel $\tilde{Y}$ d'une $Y$ compacte Kähler lisse (ou à singularités terminales) adéquate?

- Puisque l'on a une suite exacte $: K \rightarrow \pi_{1}(X \mid \Delta) \rightarrow \pi_{1}(X) \rightarrow 1$ dans laquelle $K$ est engendré par des éléments de torsion en nombre fini modulo conjugaison (mais $K$ n'est pas de type fini, en général, voir $\S .2 .2)$, les questions précédentes admettent une réponse affirmative lorsque $\pi_{1}(X \mid \Delta)$ est résiduellement fini ou admet un sousgroupe d'indice fini sans torsion, donc par exemple, lorsque ce groupe est linéaire, et en particulier, nilpotent.

Par contre, les groupes résolubles de présentation finie ne sont pas nécessairement résiduellement finis (un contre-exemple résoluble au problème du mot est donné dans [Kh 81]).

- Nous montrons ici que les résultats connus concernant les quotients résolubles des groupes de Kähler s'étendent sans changement aux groupes fondamentaux des

\footnotetext{
${ }^{1}$ Après modification adéquate de $X$ et $Y$. Cette suite reste exacte, plus généralement, dans la catégorie des orbifoldes géométriques ([Ca 07, 11.7]).
} 
orbifoldes géométriques Kählériennes. En particulier :

1. Un tel quotient est virtuellement nilpotent si (et seulement si) l'orbifolde considérée n'a pas de morphisme sur une courbe orbifolde hyperbolique (§4).

2. L'ensemble de Green-Lazarsfeld du groupe fondamental d'une telle orbifolde est réunion d'un nombre fini de translatés par des éléments de torsion de sous-tores du groupe des caractères de ce groupe $(\S 3)$.

Les démonstrations sont des réductions au cas connu où $\Delta=0$ ([GL 87], [Be 92], [Si 93], [AN 93], [Ca 01] pour l'assertion 1, et [De 06] pour l'assertion 2). La propriété cruciale utilisée dans ces réductions réside dans le fait que le noyau $K$ ci-dessus est engendré par des éléments de torsion.

- Nous montrons aussi (aux $\S 5$ et $§ 7$ ) que les ensembles de Green-Lazarsfeld et les quotients résolubles du groupe fondamental coincident essentiellement pour $(X \mid \Delta)$, pour la factorisation de Stein de son image d'Albanese, et pour sa réduction algébrique. On réduit ainsi l'étude de ces invariants au cas où $X$ est projective et où son morphisme d'Albanese est génériquement fini sur son image. Il serait intéressant de savoir si ces résultats subsistent pour l'image d'Albanese (et non seulement pour sa factorisation de Stein. Voir la question 5.2).

- Nous montrons enfin au $\S 6$ que les quotients résolubles de $\pi_{1}(X \mid \Delta)$ sont virtuellement abéliens si $(X \mid \Delta)$ est spéciale (voir [Ca 07] et le $\S 6$ pour la définition). Conjecturalement (6.1), $\pi_{1}(X \mid \Delta)$ est lui-même virtuellement abélien.

- Dans une autre direction, nous montrons aussi (au §8) que la classe de nilpotence modulo torsion des quotients nilpotents des groupes de Kähler n'est pas seulement additive dans une fibration, mais satisfait une inégalité non-archimédienne. La démonstration repose sur la structure de Hodge mixte de R. Hain sur la complétion nilpotente du groupe fondamental, et le caractère strict des morphismes de SHM, dû à $\mathrm{P}$. Deligne.

- Il serait très intéressant de savoir si les résultats précédents peuvent être étendus aux groupes fondamentaux des orbifoldes logarithmiques (ie : des variétés "quasiprojectives" ou "quasi-Kähler") en utilisant la théorie de Hodge mixte. Certains résultats sont déjà connus dans des situations particulières de ce cas ([A 98], [AN 93], [M 78]).

Remerciements : Je voudrais remercier T. Delzant pour m'avoir communiqué ses textes [De 06] et [De 07], dont les arguments du $\S 4$ sont adaptés, pour m'avoir fourni une photocopie de [BS], et signalé l'oubli d'une condition dans une version initiale du présent texte.

\section{Rappels sur les orbifoldes géométriques et leurs revêtements.}

On rappelle ici très brièvement certaines des notions relatives aux orbifoldes géométriques introduites dans [Ca 01] et [Ca 07]. On se limite à celles qui sont 
strictement indispensables pour le présent texte.

\subsection{Orbifoldes géométriques}

Si $X$ est un espace analytique complexe normal et connexe, un diviseur orbifolde $\Delta$ sur $X$ est un $\mathbb{Q}$-diviseur effectif de la forme $\Delta:=\sum_{J}\left(1-1 / m_{j}\right) \cdot D_{j}$, dans lequel les $m_{j} \geq 2$ sont, soit des entiers ${ }^{2}$, soit $+\infty$, et où les $D_{j}$ sont des diviseurs de Weil irréductibles distincts de $X$, localement finis. La réunion des $D_{j}$ est appelée le support de $\Delta$, noté $\operatorname{Supp}(\Delta)$. Le couple $(X \mid \Delta)$ est une orbifolde géométrique, dite logarithmique (resp. finie) si $m_{j}=+\infty, \forall j$ (resp. si $m_{j}<+\infty, \forall j$ ). Les orbifoldes géométriques interpolent donc lorsque $X$ est compacte, entre les cas propre (où $\Delta=0)$, et logarithmique. On dira enfin que $(X \mid \Delta)$ est lisse si $X$ est lisse et si $\operatorname{Supp}(\Delta)$ est un diviseur à croisement normaux. On dira enfin que $(X \mid \Delta)$ est Kähler si $X$ est lisse, Kählérienne, compacte et connexe.

Pour tout diviseur de Weil irréductible de $X$, on notera $m_{\Delta}(D)$ sa multiplicité dans $\Delta$, égale à $m_{j}$ si $D=D_{j}$ pour l'un (unique) des $j \in J$, et à 1 sinon.

On dira que l'orbifolde $\Delta$ sur $X$ divise l'orbifolde $\Delta_{1}$ sur $X$ si les multiplicités de $\Delta$ divisent toutes celles de $\Delta_{1}$ (ie : si pour tout $D, m_{\Delta}(D)$ divise $m_{\Delta_{1}}(D)$ ).

Un cas particulier classique est celui des orbifoldes de courbes, dans lequel $X=C$ est une courbe projective lisse et connexe, et $\Delta$ est alors la donnée d'un nombre fini de points $p_{j}$ affectés de multiplicités entières $m_{j}$. Le fibré canonique est alors le $\mathbb{Q}$ diviseur $K_{C \mid \Delta}:=K_{C}+\Delta$, dont le degré est : $\operatorname{deg}\left(K_{C \mid \Delta}\right):=2(g-1)+\sum_{j}\left(1-1 / m_{j}\right)$. On dit que $C \mid \Delta)$ est hyperbolique si $\operatorname{deg}\left(K_{C}+\Delta\right)>0$.

Un morphisme orbifolde ${ }^{3} f:(X \mid \Delta) \rightarrow\left(C \mid \Delta_{C}\right)$ d'une orbifolde géométrique sur une courbe orbifolde est une application holomorphe surjective et à fibres connexes de l'espace analytique normal connexe et compact $X$ sur la courbe projective lisse $C$ telle que pour tout $b \in S u p p\left(\Delta_{C}\right)$, si $f^{*}(b)=\sum_{k} t_{k} . E_{k}$, on a, pour tout $k: m_{\Delta_{C}}(b)$ divise $t_{k} \cdot m_{\Delta}\left(E_{k}\right)$.

Si $f: X \rightarrow C$ est un morphisme surjectif, avec $X, C$ comme précédemment, et si $(X \mid \Delta)$ est une structure orbifolde sur $X$, on définit la base orbifolde de $(f, \Delta)$ sur $C$, notée $\Delta_{f, \Delta}$ (ou simplement $\Delta_{f}$ s'il n'y a pas d'ambiguité sur $\Delta$ ), comme étant la plus petite des structures orbifoldes $\Delta_{C}$ sur $C$ telle que $f:(X \mid \Delta) \rightarrow\left(C \mid \Delta_{C}\right)$ soit un morphisme orbifolde. Explicitement : $m_{\Delta_{f}}(b)=\operatorname{pgcd}\left\{t_{k} \cdot m_{\Delta}\left(E_{k}\right)\right\}$ pour tout $b \in C$, avec les notations précédentes.

Lorsque $\operatorname{dim}(C)>1$, la définition est la même, mais en omettant les diviseurs $f$-exceptionnels de $X$.

\footnotetext{
${ }^{2}$ Il s'agit donc ici seulement de diviseurs orbifoldes entiers dans la terminologie de [Ca 07].

${ }^{3} \mathrm{Il}$ s'agit des morphismes orbifoldes divisibles dans la terminologie de [Ca 07] qui considère des morphismes et orbifoldes géométriques plus généraux.
} 
Remarquons que si $d: Y \rightarrow X$ et $f: X \rightarrow C$ sont des fibrations, et $F:=f \circ d$, alors : $\Delta_{F}$ divise $\Delta_{f}$, et $\Delta_{f}$ divise $\Delta_{f, \Delta}$ pour tout diviseur orbifolde $\Delta$ sur $X$. Ces assertions résultent $\mathrm{d} u$ fait que pour tous entiers $m_{j}, t_{j}$, on a $: \operatorname{pgcd}_{j}\left\{t_{j}\right\}$ divise $\operatorname{pgcd}_{j}\left\{t_{j} \cdot m_{j}\right\}$.

\subsection{Groupe fondamental}

On s'intéressera ici, lorsque $(X \mid \Delta)$ est lisse et Kähler, à son groupe fondamental $\pi_{1}(X \mid \Delta)$, et à la structure de son revêtement universel $(\widetilde{X \mid \Delta})$, définis ci-dessous.

Rappelons donc (voir $[\mathrm{Ca} 07, \S 11])$ que $\pi_{1}(X \mid \Delta):=\pi_{1}(X-\operatorname{Supp}(\Delta)) / L$, où $L$ est le sous-groupe normal de $\pi_{1}(X-S u p p(\Delta))$ engendré par tous les éléments de la forme $l_{j}^{m_{j}}$, où $l_{j}$ est un petit lacet entourant une fois le divideur $D_{j}$. (On omet les points-base). C'est donc un groupe de présentation finie puisque $\pi_{1}(X-S u p p(\Delta))$ est de présentation finie.

On a donc un morphisme naturel surjectif : $\pi_{1}(X \mid \Delta) \rightarrow \pi_{1}(X)$ dont le noyau $K$ est engendré par des éléments de torsion (les images dans $\pi_{1}(X)$ des éléments $l_{j}$ ci-dessus. Voir [Ca 01], lemme 1.9.9). Cette propriété sera cruciale dans la suite. Observer cependant que $K$ n'est en général, ni de torsion, ni de type fini. Une première conséquence de cette propriété est que si $r: \pi_{1}(X \mid \Delta) \rightarrow G$ est une représentation dans un groupe $G$ sans torsion, elle se factorise par $\pi_{1}(X)$.

Plus généralement, si $r: \pi_{1}(X \mid \Delta) \rightarrow G$ est une représentation dans un groupe résiduellement fini (par exemple si $G \subset G l(N, \mathbb{C})$ est linéaire), alors $r$ se factorise par un sous-groupe d'indice fini, c'est-à-dire : par $\pi_{1}\left(X^{\prime}\right)$, où $X^{\prime}$ est un revêtement étale fini de $X$. Les groupes $G$ qui sont nilpotents sont résiduellement finis, mais pas les groupes résolubles, en général.

\subsection{Revêtement universel orbifolde}

Soit $(X \mid \Delta), \Delta:=\sum_{j}\left(1-\frac{1}{m_{j}}\right) \cdot D_{j}$ une orbifolde géométrique lisse ${ }^{4}$. Elle admet (voir $[\mathrm{N}, 1.3 .15, \mathrm{p} .43])$ un revêtement universel $r: \widetilde{(X \mid \Delta)} \rightarrow(X \mid \Delta)$, c'est-à-dire un revêtement connexe et simplement connexe, normal, Galoisien de groupe $G=$ $\pi_{1}(X \mid \Delta)$, non-ramifié au-dessus du complémentaire de $\operatorname{Supp}(\Delta)$, et ramifiant audessus de chaque point lisse $x \in D_{j}$ du support de $\Delta$ à un ordre $m_{j}^{\prime}$ qui divise $m_{j}$. Un tel revêtement est unique à automorphisme de revêtement près. Les entiers $m_{j}^{\prime}$ sont également déterminés par ces conditions. L'orbifolde géométrique $\left(X \mid \Delta^{\prime}\right)$, avec $\Delta^{\prime}:=\sum_{j}\left(1-\frac{1}{m_{j}^{\prime}}\right)$. $D_{j}$ est la "réduction" de $(X \mid \Delta)$. On a, en particulier : $G=\pi_{1}(X \mid \Delta) \cong \pi_{1}\left(X \mid \Delta^{\prime}\right)$. Voir [Ca 07, §11.3] pour plus de détails.

\footnotetext{
${ }^{4}$ Les énoncés qui suivent sont en fait valables, plus généralement, lorsque les groupes fondamentaux locaux sont finis.
} 
Par exemple, si $X=C$ est une courbe projective lisse et connexe, alors $(C \mid \Delta)$ est hyperbolique si et seulement si son revêtement universel est le disque unité $\mathbb{D}$, et $(C \mid \Delta)=\mathbb{D} / G$, où $G$ est le $\pi_{1}$-orbifolde de $(C \mid \Delta)$, agissant proprement et discontinuement sur le disque unité $\mathbb{D}$. Une telle orbifolde est "réduite".

On a, de plus, une bijection naturelle entre :

1. les sous-groupes $G^{\prime}$ de $G:=\pi_{1}(X \mid \Delta)$, et :

2. Les revêtements orbifolde-étales $\left(X^{\prime} \mid \Delta^{\prime}\right) \rightarrow(X \mid \Delta)$ : ce sont ceux qui sont nonramifiés au-dessus du complémentaire de $\operatorname{Supp}(\Delta)$, et ramifient au-dessus de chaque point lisse $x \in D_{j}$ du support de $\Delta$ à un ordre $m_{j}^{\prime}$ qui divise $m_{j}$.

Par exemple, si $a:\left(C^{\prime} \mid \Delta_{C^{\prime}}\right) \rightarrow\left(C \mid \Delta_{C}\right)$ est un morphisme orbifolde-étale, alors $\left(C \mid \Delta_{C}\right)$ est hyperbolique si et seulement si $\left(C^{\prime} \mid \Delta_{C^{\prime}}\right)$ l'est.

Un tel revêtement $X^{\prime}$ a, au plus, des singularités quotient. Si $d^{\prime}: Y^{\prime} \rightarrow X^{\prime}$ est une désingularisation, elle induit donc un isomorphisme entre $\pi_{1}\left(Y^{\prime}\right)$ et $\pi_{1}\left(X^{\prime}\right)$, par [Ko 93, 7.2-7.5].

Les singularités de l'espace normal $(\widetilde{X \mid \Delta})$ sont des singularités quotient (par des groupes abéliens). Cet espace admet une désingularisation $G$-équivariante $d: \widetilde{Y} \rightarrow$ $(\widetilde{X \mid \Delta)}$.

Nous aurons besoin du :

Lemme 2.1 Soit un diagramme commutatif :

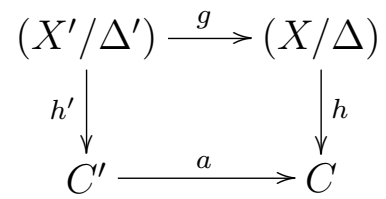

dans lequel les flèches verticales sont des fibrations sur des courbes projectives, $X$ et $X^{\prime}$ étant normaux et connexes compacts. On suppose que $g$ est orbifolde-étale. Alors, $a:\left(C^{\prime} \mid \Delta_{h^{\prime}, \Delta^{\prime}}\right) \rightarrow\left(C \mid \Delta_{h, \Delta}\right)$ est aussi orbifolde-étale.

En particulier : $\left(C \mid \Delta_{h, \Delta}\right)$ est hyperbolique si et seulement si $\left(C^{\prime} \mid \Delta_{h^{\prime}, \Delta^{\prime}}\right)$ l'est.

Démonstration : Soit $b \in C$, et $b^{\prime} \in C^{\prime}$ tel que $a\left(b^{\prime}\right)=b$. On note $r_{a}\left(b^{\prime}\right)$ l'ordre de ramification de $a$ en $b^{\prime}$. Il s'agit de montrer que $r_{a}\left(b^{\prime}\right) \cdot m_{\Delta f^{\prime}}\left(b^{\prime}\right)=m_{\Delta_{f}}(b)$.

On a : $h^{*}(b)=\sum_{k} t_{k} \cdot F_{k}$, et $g^{*}\left(\sum_{k} t_{k} \cdot F_{k}\right)=\sum_{k, h} t_{k} \cdot r_{g}\left(F_{k, h}^{\prime}\right)+\ldots$, en posant : $g^{*}\left(F_{k}\right)=\sum_{h} r_{g}\left(F_{k, h}^{\prime}\right) \cdot F_{k, h}^{\prime}+\ldots$, en désignant par $F_{k, h}^{\prime}$ les composantes irréductibles de $g^{-1}\left(F_{k}\right)$ contenues dans $\left(h^{\prime}\right)^{-1}\left(b^{\prime}\right)$, et par $r_{g}\left(F_{k, h}^{\prime}\right)$ l'ordre de ramification de $g$ le long de $F_{k, h}^{\prime}$.

Par ailleurs : $r_{a}\left(b^{\prime}\right) \cdot\left(h^{\prime}\right)^{*}\left(b^{\prime}\right)=r_{a}\left(b^{\prime}\right) \cdot \sum_{k, h} \cdot t_{k, h}^{\prime} \cdot F_{k, h}^{\prime}=\sum_{k, h} t_{k} \cdot r_{g}\left(F_{k, h}^{\prime}\right) \cdot F_{k, h}^{\prime}$, puisque $h \circ g=a \circ h^{\prime}$.

Donc : $r_{a}\left(b^{\prime}\right) \cdot t_{k, h}^{\prime}=t_{k} \cdot r_{g}\left(F_{k, h}^{\prime}\right), \forall k, h$.

Puisque $g$ est orbifolde-étale, on a aussi : $m_{\Delta^{\prime}} \cdot r_{g}\left(F_{k, h}^{\prime}\right)=m_{\Delta}\left(F_{k}\right), \forall k, h$.

Par définition : $r_{a}\left(b^{\prime}\right) \cdot m_{\Delta_{f}^{\prime}}\left(b^{\prime}\right)=\operatorname{pgcd}_{(k, h)}\left\{r_{a}\left(b^{\prime}\right) \cdot t_{k, h}^{\prime} \cdot m_{\Delta^{\prime}}\left(F_{k, h}^{\prime}\right)\right\}=$

$=\operatorname{pgcd}_{(k, h}\left\{t_{k} \cdot r_{g}\left(F_{k, h}^{\prime}\right) \cdot m_{\Delta^{\prime}}\left(F_{k, h}^{\prime}\right)\right\}=\operatorname{pgcd}_{(k, h}\left\{t_{k} \cdot m_{\Delta}\left(F_{k}\right)\right\}=m_{\Delta_{f}}(b)$ 


\section{Ensembles de Green-Lazarsfeld des orbifoldes géométriques.}

\subsection{Ensembles de Green-Lazarsfeld : généralités.}

Soit $G$ un groupe de type fini, $D G$ son groupe dérivé (engendré par ses commutateurs), $G_{a b}=G / D G$ son abélianisé, et $H^{1}(G, \mathbb{C}):=\operatorname{Hom}(G, \mathbb{C})=\operatorname{Hom}\left(G_{a b}, \mathbb{C}\right)$.

Le groupe des caractères complexes (multiplicatifs) de $G$ est $\hat{G}:=\operatorname{Hom}\left(G, \mathbb{C}^{*}\right)$. On notera $\chi$ un tel caractère, et $\mathbb{C}_{\chi}$ le $G$-module dont le groupe additif est $\mathbb{C}$, muni de l'action : $g \cdot u:=\chi(g) . u$, pour $(u, g) \in \mathbb{C} \times G$.

On note $\Sigma^{1}(G) \subset \hat{G}$ l'ensemble de Green-Lazarsfeld de $G$, constitué des caractères $\chi: G_{a b} \rightarrow \mathbb{C}^{*}$ tels que $H^{1}\left(G, \mathbb{C}_{\chi}\right) \neq 0$. La suite spectrale de Hochschild-Serre fournit un isomorphisme naturel $H^{1}\left(G, \mathbb{C}_{\chi}\right) \cong \operatorname{Hom}_{G}\left((D G)_{a b}, \mathbb{C}_{\chi}\right), G$ agissant sur $(D G)_{a b}$ par conjugaison, et sur $\mathbb{C}$ par multiplication par $\chi([\operatorname{Be} 92,4.2])$.

Un morphisme de groupes surjectif $q: G \rightarrow Q$ induit des injections $q^{*}: \hat{Q} \rightarrow \hat{G}$ et $\Sigma^{1}(Q) \rightarrow \Sigma^{1}(G)$.

Si $(X \mid \Delta)$ est une orbifolde géométrique finie, lisse et (compacte) Kähler, et $G:=$ $\pi_{1}(X \mid \Delta), H:=\pi_{1}(X)$, on note $f: G \rightarrow H$ le morphisme de groupes surjectif naturel, de noyau $K$. On posera : $\Sigma^{1}(X \mid \Delta):=\Sigma^{1}(G) \subset \hat{G}=H^{1}\left(X, \mathbb{C}^{*}\right)$. On a une inclusion évidente $f^{*}: \hat{H} \rightarrow \hat{G}$ dont le conoyau est fini, puisque $K$ est engendré par des éléments de torsion, et que $G$ est de type fini.

\subsection{Ensembles de Green-Lazarsfeld orbifoldes.}

Théorème 3.1 Soit $(X \mid \Delta)$ une orbifolde géométrique finie, lisse et Kähler, et $\Sigma^{1}(X \mid \Delta) \subset H^{1}\left(X, \mathbb{C}^{*}\right)$ son ensemble de Green-Lazarsfeld. Alors :

1. $\Sigma^{1}(X \mid \Delta)$ est une réunion finie de translatés de sous-tores par des éléments de torsion.

2. Si $S$ est une composante connexe de dimension strictement positive de $\Sigma^{1}(X \mid \Delta)$, il existe un morphisme orbifolde $f$ de $(X \mid \Delta)$ sur une courbe orbifolde hyperbolique $\left(C \mid \Delta_{C}\right)$, et un élément $\tau$ de torsion dans $\hat{G}$ tels que $S=\tau \cdot f^{*}\left(\widehat{\pi_{1}(C)}\right)$. (Plus précisément ${ }^{5}$ : $S$ est l'une des composantes de $f^{*}\left(\Sigma^{1}\left(C \mid \Delta_{C}\right)\right)$.

La situation est donc l'exact analogue du cas où $\Delta=0$ traité dans [GL 87], [Be 92], [Si 93] et [Ca 01]. La démonstration consiste d'ailleurs à se ramener à ce cas particulier par des arguments dont certains sont analogues à ceux utilisés dans [Ca 01, (1.9.3-1.11.8, pp. 603-607) ] pour déduire le cas Kähler du cas projectif.

Démonstration Posons $G:=\pi_{1}(X \mid \Delta)$, et $H:=\pi_{1}(X)$. Le morphisme de groupes naturel surjectif $f: G \rightarrow H$ a un noyau $K$ engendré par des éléments

\footnotetext{
${ }^{5}$ Voir 3.8 pour un énoncé plus complet.
} 
de torsion (voir 2.2). Il induit donc un morphisme de groupes (abéliens) surjectif $(D f)_{a b}:(D G)_{a b} \rightarrow(D H)_{a b}$.

Supposons tout d'abord que $K \subset D G$. La conclusion est alors immédiate :

En effet, on a (voir, par exemple, [Be 92, prop. 4.1]) : $H^{1}\left(G, \mathbb{C}_{\chi}\right)=$ $H_{o m}\left((D G)_{a b}, \mathbb{C}_{\chi}\right),(D G)_{a b}$ étant considéré comme un $G$-module pour l'action par conjugaison de $G$ sur lui-même. L'image de $K$ dans $(D G)_{a b}$ est de torsion, puisque $K$ est engendré par des éléments de torsion, et $(D G)_{a b}$ abélien. Puisque $\mathbb{C}_{\chi}$ est un groupe (additif) sans torsion, si $0 \neq u \in \operatorname{Hom}_{G}\left((D G)_{a b}, \mathbb{C}_{\chi}\right)$, alors $u$ s'annule sur $K$. On conclut alors par le lemme 3.2 élémentaire suivant, appliqué à $K:=N$ :

Lemme 3.2 ([Ca 01, 1.11.3]) Soit $\chi \in \Sigma^{1}(G)$, et $u \in H_{\text {om }}\left((D G)_{a b}, \mathbb{C}_{\chi}\right)$. Soit $N \subset D G$ un sous-groupe normal de $D G$ tel que u s'annule sur $N$. Alors :

1. Il existe $\chi^{\prime} \in H^{1}(G / N, \mathbb{C})$ tel que $\chi=q^{*}\left(\chi^{\prime}\right)$, si $q: G \rightarrow Q:=G / N$ est le quotient.

2. Il existe $v \in \operatorname{Hom}_{Q}\left((D Q)_{a b}, \mathbb{C}_{\chi^{\prime}}\right)$ tel que $u=q^{*}(v)$ (dans un sens fonctoriel évident)

Démonstration de 3.2 : Il suffit de vérifier l'existence de $\chi^{\prime}$, c'est-à-dire que $\chi(k)=1$ pour $k \in N$. Par hypothèse, il existe $x \in \mathbb{C}$ tel que $u(x) \neq 0$. On a : $u\left(k x k^{-1}\right)=\chi(k) \cdot u(x)$. Mais $u\left(k x k^{-1}\right)=u\left(k\left(x k^{-1} x^{-1}\right) x\right)=u(k)+u\left(x k^{-1} x^{-1}\right)+$ $u(x)=0+0+u(x)$, puisque $\left(x k^{-1} x^{-1}\right) \in N$ par normalité de $N$ dans $D G$. Donc: $\chi(k) \cdot u(x)=u(x)$, et $\chi(k)=1$, puisque $u(x) \neq 0$

Nous allons maintenant nous affranchir de l'hypothèse ${ }^{6}$ précédente $: K \subset D G$ en passant à un revêtement étale abélien adéquat. La première étape repose sur le lemme 3.3 suivant :

Lemme 3.3 (Voir $[C a$ 01, 1.9.8]) Avec les notations précédentes, on a :

1. $K \cap D G$ est d'indice fini dans $D G$.

2. Il existe un sous-groupe $G^{\prime} \subset G$ normal et d'indice fini, avec $G / G^{\prime}$ abélien, tel que : $K \cap G^{\prime} \subset D G$.

Démonstration de 3.3 : L'application composée $K \rightarrow G \rightarrow G_{a b}$ a pour noyau $K \cap D G$, et une image $T$ finie, puisque $K$ est engendré par des éléments de torsion, et $G_{a b}$ abélien de type fini. D'où l'assertion 1 .

Soit $G$ " $\subset G_{a b}$ un supplémentaire dans $G_{a b}$ (donc sans torsion) du sous-groupe de torsion $\operatorname{Tors}\left(G_{a b}\right) \supset T$. C'est-à-dire que l'on a : $G_{a b}=G^{\prime \prime} \oplus \operatorname{Tors}\left(G_{a b}\right)$, et en particulier : $G^{\prime \prime} \cap T=\{1\}$. On définit alors $G^{\prime}$ comme étant l'image réciproque de

\footnotetext{
${ }^{6} \mathrm{Si} K$ (même métabélien et virtuellement abélien) est engendré par des éléments de torsion, il n'en est pas en général de même de ses sous-groupes d'indice fini. Par exemple, si $A$ est abélien additif sans torsion, et $K$ le produit semi-direct de $A$ avec $\mathbb{Z}_{2}$ opérant sur $A$ par : $u(a):=(-1)^{u} . a$ pour $(u, a) \in \mathbb{Z}_{2} \times A$, alors tout sous-groupe propre de $K$ est contenu dans $A$, et donc sans torsion. L'argument utilisé lorsque $K \subset D G$ ne s'applique pas. La conclusion $\Sigma^{1}(G)=f^{*}\left(\Sigma^{1}(H)\right)$ est d'ailleurs fausse, en général.
} 
$G$ " dans $G$. Alors : $K \cap G \subset D G$. En effet : l'image de $G^{\prime}$ dans $G_{a b}$ est sans torsion, tandis que celle de $K$ est finie

- Nous choisissons et fixons maintenant un sous-groupe normal et d'indice fini $G^{\prime} \subset G$ comme dans le lemme précédent. On note $r^{*}: \widehat{G} \rightarrow \widehat{G^{\prime}}$ la restriction naturelle (de noyau fini).

Lemme $3.4 r^{*}\left(\Sigma^{1}(\widehat{G})\right) \subset \Sigma^{1}\left(G^{\prime}\right) \cup \mathcal{T}$, où $\mathcal{T} \subset \widehat{G^{\prime}}$ est un ensemble fini composé d'éléments de torsion.

Démonstration : Si $0 \neq u \in \operatorname{Hom}_{G}\left(D G_{a b}, \mathbb{C}_{\chi}\right)$ est tel que $0=r^{*}(u) \in$ $\operatorname{Hom}_{G^{\prime}}\left(\left(D G^{\prime}\right)_{a b}, \mathbb{C}_{r^{*}}(\chi)\right)$, alors $u$ s'annule sur $D G^{\prime}$, et définit donc un élément non nul de $\operatorname{Hom}_{G}\left(\left(D G / D G^{\prime}\right)_{a b}, \mathbb{C}_{\chi}\right)$.

Le diagramme commutatif suivant (où les applications sont les applications naturelles) montre que le groupe abélien $\left(D G / D G^{\prime}\right)_{a b}$ est de type fini (comme $\mathbb{Z}$-module), puisqu'il a un sous-groupe d'indice fini isomorphe à $S$.

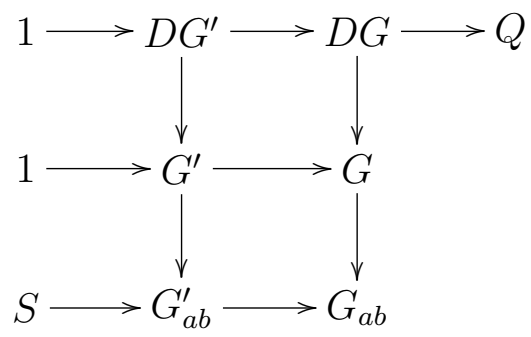

Les caractères $\chi$ de $G$ tels que $0 \neq H_{\text {om }}\left(\left(D G / D G^{\prime}\right)_{a b}, \mathbb{C}_{\chi}\right)$ sont donc en nombre fini. Montrons maintenant qu'ils sont de torsion. En effet : le groupe $G^{\prime} / D G^{\prime}$ est abélien. Donc $G^{\prime}$ agit trivialement sur $\left.\left(D G \cap G^{\prime}\right) / D G^{\prime}\right)$, qui est d'indice fini dans $\left(D G / D G^{\prime}\right)_{a b}$. L'image de $G_{a b}$ dans $\left.\operatorname{Aut}\left(D G \cap G^{\prime}\right) / D G^{\prime}\right)$ est donc finie, et il en est donc de même de son image dans $\operatorname{Aut}\left(\left(D G / D G^{\prime}\right)_{a b}\right.$, puisque le noyau de l'application de restriction : Aut $\left(\left(D G / D G^{\prime}\right)_{a b} \rightarrow \operatorname{Aut}\left(\left(D G \cap G^{\prime}\right) / D G^{\prime}\right)\right.$ est fini. Les charactères $\chi$ de $G$ intervenant dans la représentation linéaire $G_{a b} \rightarrow G L\left(\left(D G / D G^{\prime}\right)_{a b} \otimes_{\mathbb{Z}} \mathbb{C}\right)$ sont donc bien de torsion, et en nombre fini

- Soit $g:\left(X^{\prime} \mid \Delta^{\prime}\right) \rightarrow(X \mid \Delta)$ le revêtement orbifolde-étale fini défini par l'inclusion $G^{\prime} \subset G$ de 3.3 ci-dessus (voir 2.3 ci-dessus). Soit $h^{\prime}: G^{\prime} \rightarrow H^{\prime}:=\pi_{1}\left(X^{\prime}\right)$ le morphisme de groupes naturel. Son noyau $K^{\prime}$ est donc engendré par des éléments de torsion. De plus, $K^{\prime} \subset\left(K \cap G^{\prime}\right) \subset D G$.

Par le lemme 3.4 ci-dessus, quitte à négliger un nombre fini de caractères de torsion de $G$, on peut supposer que si $\chi \in \widehat{G}$, et si $0 \neq u \in \operatorname{Hom}_{G}\left(D G_{a b}, \mathbb{C}_{\chi}\right)$, alors $0 \neq r^{*}(u) \in \operatorname{Hom}_{G^{\prime}}\left(\left(D G^{\prime}\right)_{a b}, \mathbb{C}_{r^{*}(\chi)}\right)$.

Par le lemme 3.2 ci-dessus, $r^{*}(\chi)=\left(h^{\prime}\right)^{*}\left(\chi^{\prime}\right)$, pour $\chi^{\prime} \in \widehat{H}^{\prime}$, et $r^{*}(u)=\left(h^{\prime}\right)^{*}\left(u^{\prime}\right)$, pour $0 \neq u^{\prime} \in H^{1}\left(H^{\prime}, \mathbb{C}_{\chi^{\prime}}\right)$. Donc $\chi^{\prime} \in \Sigma^{1}\left(X^{\prime}\right)$.

Soit $d^{\prime}: Y^{\prime} \rightarrow X^{\prime}$ une désingularisation Kählérienne de $X^{\prime}$ (qui est un revêtement ramifié de $X$ à singularités quotient). Donc $d_{*}^{\prime}: \pi_{1}\left(Y^{\prime}\right) \rightarrow \pi_{1}\left(X^{\prime}\right)$ est un isomorphisme, et $\Sigma^{1}\left(Y^{\prime}\right)=\Sigma^{1}\left(X^{\prime}\right)$. 
- Distinguons deux cas :

1. $\chi$ est isolé dans $\Sigma^{1}(X \mid \Delta)$. Alors $\chi^{\prime}$ est isolé dans $\Sigma^{1}\left(Y^{\prime}\right)$, donc de torsion, par [Ca 01] (qui réduit le cas Kähler au cas projectif, établi par C. Simpson dans [Si 93]). Donc $\chi$ est aussi de torsion.

2. $\chi$ n'est pas isolé dans $\Sigma^{1}(X \mid \Delta)$. Donc $\chi^{\prime}$ n'est pas isolé dans $\Sigma^{1}\left(Y^{\prime}\right)$ (par le lemme 3.4). Par [Be 92, 3.5] reformulé dans la terminologie orbifolde, pour toute composante irréductible de $\Sigma^{1}\left(Y^{\prime}\right)$ contenant $r^{*}(\chi)$, il existe un morphisme orbifolde $f^{\prime}: X^{\prime} \rightarrow\left(C^{\prime} \mid \Delta_{f^{\prime}}\right)$ tel que la composante irréductible considérée de $\Sigma^{1}\left(Y^{\prime}\right)$ contenant $r^{*}\left(\chi^{\prime}\right)$ soit égale à une composante irréductible (ou connexe) de $\left(f^{\prime}\right)^{*}\left(\Sigma^{1}\left(M^{\prime}\right)\right.$, si $M^{\prime}:=\pi_{1}\left(C^{\prime} \mid \Delta_{f^{\prime}}\right)$. Une telle composante est donc réunion des $\tau \cdot f^{*}\left(\gamma^{\prime}\right)$ pour $\gamma^{\prime} \in$ $H^{1}\left(C^{\prime}, \mathbb{C}^{*}\right)$, et $\tau$ fixé, de torsion dans $\widehat{H^{\prime}}$. Fixons une telle composante.

- On construit maintenant un diagramme commutatif, avec $C$ une courbe, $f$ et $a$ surjectifs :

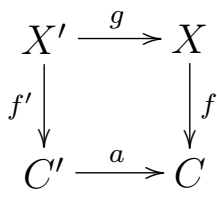

Pour cela, considérons le diagramme commutatif :

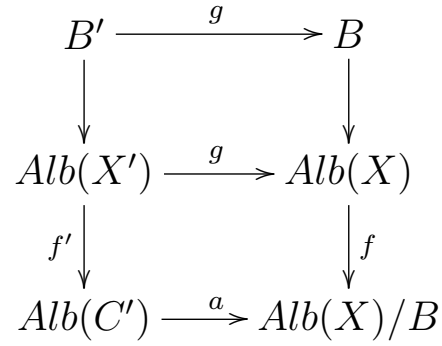

Dans lequel on utilise les mêmes lettres pour désigner une application, l'une de ses restrictions, et l'application induite au niveau des variétés d'Albanese. De plus, $B^{\prime}$ désigne le noyau (qui est connexe) du morphisme $f^{\prime}$, et $B:=g\left(B^{\prime}\right)$.

Dans ce diagramme, $B \neq A l b(X)$. En effet : $\chi^{\prime}=\left(f^{\prime}\right)^{*}\left(\gamma^{\prime}\right)$ est trivial sur le noyau $\pi_{1}\left(B^{\prime}\right)$, et $\chi$ est donc trivial sur $\pi_{1}(B)$, puisque $g^{*}(\chi)=\chi^{\prime}$. Si $B=\operatorname{Alb}(X)$, $\chi$ est donc de torsion, contrairement à notre hypothèse. (Remarquer que $\widehat{\pi_{1}(X)}$ et $\pi_{1}(\widehat{\operatorname{Alb}(X))}$ ont la même composante neutre).

Donc : $C:=a\left(C^{\prime}\right)$ est une courbe irréductible de $A l b(X) / B$, puisque $C^{\prime}$ engendre $A l b\left(C^{\prime}\right)$, de sorte que $C$ engendre $A l b(X) / B \neq\{0\}$. La commutativité du diagramme précédent montre que $F:=f \circ \alpha_{X}: X \rightarrow C$ est surjective, avec $F \circ g=a \circ f^{\prime}$ : $X^{\prime} \rightarrow C$. (On a normalisé $C$, et encore noté $a$ la restriction de $a$ à $C^{\prime}$, enfin $\alpha_{X}$ est le morphisme d'Albanese de $X)$.

- On déduit des morphismes $f^{\prime}: X^{\prime} \rightarrow C^{\prime}$ et $F: X \rightarrow C$ le diagramme commutatif suivant de morphismes orbifoldes, dans lequel $g$ est, par construction, orbifolde- 
étale :

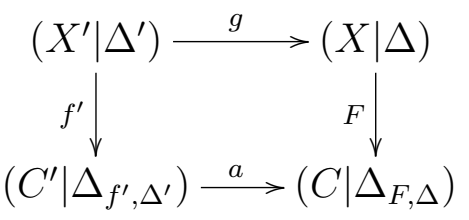

On déduit alors du lemme 2.1 que $a:\left(C^{\prime} \mid \Delta_{f^{\prime}, \Delta^{\prime}}\right) \rightarrow\left(C \mid \Delta_{F, \Delta}\right)$ est orbifoldeétale, et donc que $\left(C \mid \Delta_{F, \Delta}\right)$ est une courbe orbifolde hyperbolique si $\left(C^{\prime} \mid \Delta_{f^{\prime}, \Delta^{\prime}}\right)$ l'est. Vérifions cette dernière propriété.

Soit $F^{\prime}:=f^{\prime} \circ d^{\prime}: Y^{\prime} \rightarrow C^{\prime}$; nous savons que $\left(C^{\prime} \mid \Delta_{F^{\prime}}\right)$ est hyperbolique. Donc $\left(C^{\prime} \mid \Delta_{f^{\prime}}\right)$ est a fortiori hyperbolique, puisque $\Delta_{F^{\prime}}$ divise évidemment $\Delta_{f^{\prime}}$ (voir la dernière remarque de la section 2.1). Enfin, $\Delta_{f^{\prime}}$ divise $\Delta_{f^{\prime}, \Delta^{\prime}}$ (également par 2.1), ce qui établit l'hyperbolicité de $\left(C^{\prime} \mid \Delta_{f^{\prime}, \Delta^{\prime}}\right)$.

- Le théorème 3.1 résulte alors des lemmes 3.5 et 3.6 ci-dessous :

Lemme 3.5 Posons $M:=\pi_{1}\left(C \mid \Delta_{C}\right)$, avec $\Delta_{C}:=\Delta_{F, \Delta}$, et $M^{\prime}:=\pi_{1}\left(C^{\prime} \mid \Delta_{f^{\prime}}\right)$.

Si $\chi \in \Sigma^{1}(G)$ n'est pas isolé dans $\Sigma^{1}(G)$, alors :

1. $r^{*}(\chi)=\left(F^{\prime}\right)^{*}\left(\gamma^{\prime}\right), \gamma^{\prime} \in \Sigma^{1}\left(M^{\prime}\right) \subset \Sigma^{1}\left(M^{\prime \prime}\right)$.

2. $H^{1}\left(G^{\prime}, \mathbb{C}_{r^{*}}(\chi)\right)=\left(F^{\prime}\right)^{*}\left(H^{1}\left(M^{\prime}, \mathbb{C}_{\gamma^{\prime}}\right)\right)$.

3. il existe $\gamma \in \Sigma^{1}(M)$ tel que $\chi=F^{*}(\gamma)$, et $H^{1}\left(G, \mathbb{C}_{\chi}\right) \subset F^{*}\left(H^{1}\left(M, \mathbb{C}_{\gamma}\right)\right)$.

Démonstration : Assertion 1. Nous avons vu que $r^{*}(\chi)=\left(h^{\prime}\right)^{*}\left(\chi^{\prime}\right)$ pour un $\chi^{\prime} \in \Sigma^{1}\left(H^{\prime}\right)$. Par [Be 92], $\Sigma^{1}\left(H^{\prime}\right)=\left(f^{\prime}\right)^{*}\left(\Sigma^{1}\left(M^{\prime}\right)\right)$. D'où l'assertion, puisque $\left(F^{\prime}\right)^{*}:=$ $\left(h^{\prime}\right)^{*} \circ\left(f^{\prime}\right)^{*}$.

Assertion 2. Nous allons utiliser plusieurs des arguments de [Be 92]. Posons : $\chi^{\prime}:=$ $r^{*}(\chi)$. Nous avons tout d'abord une suite exacte courte (voir [Be 92, démonstration de la prop.3.5]) :

$$
0 \rightarrow E_{2}^{1,0}\left(X^{\prime}, \chi^{\prime}\right) \rightarrow H^{1}\left(G^{\prime}, \mathbb{C}_{\chi^{\prime}}\right) \rightarrow E_{2}^{0,1}\left(X^{\prime}, \chi^{\prime}\right),
$$

dans laquelle $E_{2}^{1,0}\left(X^{\prime}, \chi^{\prime}\right)$ est l'homologie du complexe :

$$
H^{0}\left(X^{\prime}, L^{\prime}\right) \rightarrow H^{0}\left(X^{\prime}, \Omega_{X^{\prime}}^{1} \otimes L^{\prime}\right) \rightarrow H^{0}\left(X^{\prime}, \Omega_{X^{\prime}}^{2} \otimes L^{\prime}\right),
$$

tandis que $E_{2}^{0,1}\left(X^{\prime}, \chi^{\prime}\right)$ est le noyau de la flèche :

$$
H^{1}\left(X^{\prime}, L^{\prime}\right) \rightarrow H^{1}\left(X^{\prime}, \Omega_{X^{\prime}}^{1} \otimes L^{\prime}\right),
$$

dans lesquels les flèches sont données par $\wedge \omega$, tandis que, si $\mathcal{L}^{\prime}$ est le fibré en droites plat sur $X^{\prime}$ donné par le caractère $\chi^{\prime}$, dont les sections locales sont constantes, alors $L^{\prime}$ désigne le fibré en droites holomorphe défini par : $L^{\prime}:=\mathcal{L}^{\prime} \otimes_{\mathbb{C}} \mathcal{O}_{X^{\prime}}$. Enfin, $\omega^{\prime}$ est une 1-forme holomorphe non-nulle sur $C^{\prime}$.

On a donc : $H^{1}\left(G^{\prime}, \mathbb{C}_{\chi^{\prime}}\right)=H^{1}\left(X^{\prime}, \mathcal{L}^{\prime}\right)$. 
Par fonctorialité, nous avons un diagramme commutatif :

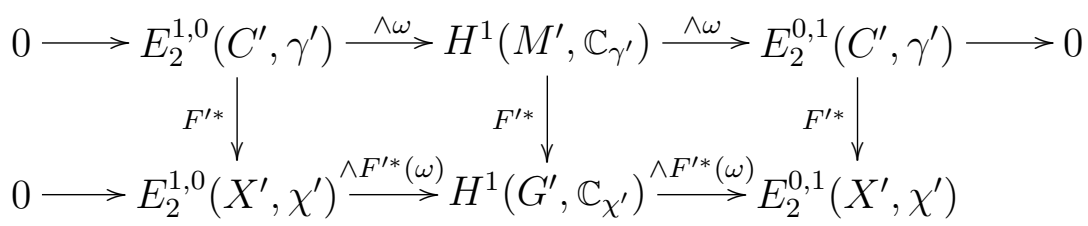

Nous voulons montrer que la flèche verticale du milieu est bijective. Il suffit de montrer que les deux autres flèches verticales extrêmes le sont. Pour celle de gauche, ceci résulte des arguments donnés dans la démonstration de [Be 92, Prop. 2.1]. Pour la flèche de droite, ceci résulte du fait que $E_{2}^{0,1}\left(X^{\prime}, \chi^{\prime}\right)$ est le conjugué de $E_{2}^{1,0}\left(X^{\prime},\left(\chi^{\prime}\right)^{-1}\right)$, ce qui est démontré (mais non formulé) dans la preuve de [Be 92 , Prop. 3.5].

Notons que les arguments qui précèdent impliquent la surjectivité de la flèche :

$$
\wedge F^{\prime *}(\omega): H^{1}\left(G^{\prime}, \mathbb{C}_{\chi^{\prime}}\right) \rightarrow E_{2}^{0,1}\left(X^{\prime}, \chi^{\prime}\right) .
$$

Assertion 3. Soit en effet $u \in \operatorname{Hom}_{G}\left(D G, \mathbb{C}_{\chi}\right)$, alors $r^{*}(u)$ ne s'annule pas sur $D G^{\prime}$, puisque $\chi$ n'est pas isolé dans $\Sigma^{1}(G)$. Puisque, par l'assertion 2 précédente, $r^{*}(u)=\left(F^{\prime}\right)^{*}(v), v \in H^{1}\left(C^{\prime}, \mathbb{C}_{\gamma^{\prime}}\right), u$ s'annule sur $\Phi^{\prime}:=\pi_{1}\left(F^{\prime}\right), F^{\prime}$ étant une fibre lisse de $f^{\prime}: X^{\prime} \rightarrow C^{\prime}$. Donc $u$ s'annule sur $g_{*}\left(\Phi^{\prime}\right)$, qui est un sous-groupe d'indice fini de $\Phi:=\pi_{1}(F), F:=g\left(F^{\prime}\right)$ est une fibre lisse de $f: X \rightarrow C$. Puisque le groupe (additif) $\mathbb{C}_{\chi}$ est sans torsion, $u$ s'annule aussi sur $\Phi$, et même sur $\Phi_{\Delta}:=\pi_{1}\left(F \mid \Delta_{F}\right)$. On a noté $\Delta_{F}:=\Delta_{\mid F}$ la restriction du diviseur $\Delta$ à une fibre générique $F$ de $f$. La dernière assertion provient de ce que le noyau du morphisme de groupes naturel $\Phi_{\Delta} \rightarrow \Phi$ est engendré par des éléments de torsion.

On dispose enfin ([Ca 07, prop.11.7]) d'une suite exacte de groupes (car tout morphisme sur une courbe est net) induite par $f_{*}$ :

$$
\Phi_{\Delta} \rightarrow G \rightarrow M \rightarrow 1
$$

Le lemme 3.2 montre alors que $\chi=F^{*}(\gamma)$, pour $\gamma \in \widehat{M}$. La seconde assertion de 3 est évidente

Lemme 3.6 Soit $C$ une courbe projective lisse et connexe de genre $g(C) \geq 1$. Soit $\Delta_{C}$ une structure orbifolde sur $C$ telle que $\left(C \mid \Delta_{C}\right)$ soit hyperbolique. Soit $M:=$ $\pi_{1}\left(C \mid \Delta_{C}\right)$. Alors : $\Sigma^{1}(M)=\widehat{M}$ si $g(C) \geq 2$, et $\Sigma^{1}(M)=\widehat{M}-\widehat{M}^{0}$ si $g(C)=1$. On désigne ici par $\widehat{M}^{0}=H^{1}\left(C, \mathbb{C}^{*}\right)$ la composante neutre de $\widehat{M}$.

Démonstration : Cet énoncé pourrait être déduit des arguments de [Be 92, $\S 1$ et Prop. 3.5]. Nous en donnons une démonstration dans l'optique orbifolde. Il existe un revêtement ramifié fini Galoisien naturel $a: C^{\prime} \rightarrow\left(C \mid \Delta_{C}\right)$ de groupe 
abélien $T:=$ Tor sion $\left(M_{a b}\right.$ ), qui est orbifolde-étale (voir aussi [Be 92, Prop. 1.9]). La propriété signifie, en particulier, que pour chaque point $p \in C$, et tout point $p^{\prime} \in C^{\prime}$ tel que $a\left(p^{\prime}\right)=p$, l'ordre de ramification de $a$ en $p^{\prime}$ est égal à la multiplicité de $p$ dans $\left.\Delta_{C}\right)$.

On a donc une suite exacte de groupes :

$$
1 \rightarrow M^{\prime} \rightarrow M \rightarrow T \rightarrow 1
$$

où : $M^{\prime}:=\pi_{1}\left(C^{\prime}\right)$, et où $T$ est le groupe de Galois du revêtement $a$. Soit $\chi \in \widehat{M}$, non de torsion. On applique la suite spectrale de Hochschild-Serre à la suite exacte précédente et au $M$-module $\mathbb{C}_{\chi}$ précédent. La suite exacte à 5 termes associée fournit en particulier :

$\left.0 \rightarrow H^{1}\left(T, H^{0}\left(M^{\prime}, \mathbb{C}_{\chi}\right)\right) \rightarrow H^{1}\left(M, \mathbb{C}_{\chi}\right)\right) \rightarrow H^{0}\left(T, H^{1}\left(M^{\prime}, \mathbb{C}_{\chi}\right)\right) \rightarrow H^{2}\left(T, H^{0}\left(M^{\prime}, \mathbb{C}_{\chi}\right)\right)$

Si la restriction à $M^{\prime}$ de $\chi$ est triviale, alors $\chi$ est de torsion. Il nous suffit donc de considérer le cas où la restriction à $M^{\prime}$ de $\chi$ est non triviale. Alors : $H^{0}\left(M^{\prime}, \mathbb{C}_{\chi}\right)=0$. Donc $\left.H^{1}\left(M, \mathbb{C}_{\chi}\right)\right) \cong H^{0}\left(T, H^{1}\left(M^{\prime}, \mathbb{C}_{\chi}\right)\right)=H^{1}\left(M^{\prime}, \mathbb{C}_{\chi}\right)^{T}$, sousgroupe des $T$-invariants de $H^{1}\left(M^{\prime}, \mathbb{C}_{\chi}\right)$.

Soit $\mathcal{L}^{\prime}$ le fibré plat sur $C^{\prime}$ défini par le caractère $\chi$. On a donc: $H^{1}\left(M^{\prime}, \mathbb{C}_{\chi}\right)=$ $H^{1}\left(C^{\prime}, \mathcal{L}^{\prime}\right)$. On note aussi $L^{\prime}:=\mathcal{L}^{\prime} \otimes \mathcal{O}_{\mathcal{C}^{\prime}}$ le fibré en droites holomorphe induit. La suite exacte longue de cohomologie associée à la suite exacte de faisceaux sur $C^{\prime}: 0 \rightarrow \mathcal{L}^{\prime} \rightarrow L^{\prime} \rightarrow \Omega_{C^{\prime}}^{1} \otimes L^{\prime} \rightarrow 0$ fournit la suite exacte courte autoduale : $0 \rightarrow H^{0}\left(C^{\prime}, \Omega_{C^{\prime}}^{1} \otimes L^{\prime}\right) \rightarrow H^{1}\left(C^{\prime}, \mathcal{L}^{\prime}\right) \rightarrow H^{1}\left(C^{\prime}, L^{\prime}\right) \rightarrow 0$.

Il existe des fibrés $\mathcal{L}$ et $L$ sur $C$ tels que $\mathcal{L}^{\prime}=a^{*}(\mathcal{L})$, et $L^{\prime}=a^{*}(L)$, et on a une action linéaire naturelle de $T$, groupe de Galois du revêtement $C^{\prime} / C$ sur les fibrés $\mathcal{L}^{\prime}$ et $L^{\prime}$, compatibles avec l'action de revêtement.

Ces actions induisent des actions de $T$ sur les cohomologies, compatibles avec la suite exacte courte précédente. Il nous suffit donc de montrer que $H^{1}\left(C^{\prime}, L^{\prime}\right)^{T} \neq 0$ pour tout $\chi$ si si $g(C) \geq 2$, et pour tout $\chi \notin H^{1}\left(C, \mathbb{C}^{*}\right)$ si $g=1$. On a donc : $H^{1}\left(C^{\prime}, L^{\prime}\right)=H^{1}\left(C^{\prime}, a^{*}(L)\right)=H^{1}\left(C, L \otimes a_{*}\left(\mathcal{O}_{C^{\prime}}\right)\right)=H^{1}\left(C, L \otimes\left[\mathcal{O}_{C} \oplus_{0 \neq t \in \hat{T}} L_{t}\right]\right)$, où $L_{t}$ est le sous-fibré "propre" pour l'action de $t \in \hat{T}$. Donc $L_{t}$ est un fibré en droites holomorphe de degré strictement négatif pour $t \neq 0$, et sur lequel l'action de $T$ se fait donc par multiplication par $t$. On en déduit immédiatemment la non-nullité de $H^{1}\left(C^{\prime}, L^{\prime}\right)^{T}$ exactement dans les conditions de l'énoncé

Remarque 3.7 Dans la situation de 3.6, si $\Delta_{C}=\sum_{j}\left(1-\frac{1}{m_{j}}\right) \cdot\left\{p_{j}\right\}$, le groupe $T$ est isomorphe au sous-groupe $T(\Delta)$ de $\oplus_{j} \mathbb{Z} / m_{j}$ constitué des familles de classes $\left(\bar{k}_{j}\right)_{j}$ telles que $\sum_{j} \frac{k_{j}}{m_{j}}$ soit entier. (Voir [Be 92, 1.5]).

On peut reformuler plus précisément les résultats obtenus :

Théorème 3.8 Soit $(X \mid \Delta)$ une orbifolde géométrique finie, lisse et Kähler. Alors : 
1. $\Sigma^{1}(X \mid \Delta)$ est une réunion finie de translatés de sous-tores par des éléments de torsion.

2. il existe un nombre fini de morphismes orbifoldes $f_{j}$ de $(X \mid \Delta)$ sur des courbes orbifoldes hyperboliques $\left(C_{j} \mid \Delta_{C_{j}}\right)$ tels que la réunion des composantes irréductibles de dimension strictement positive de $\Sigma^{1}(X \mid \Delta)$ soit la réunion des $\left(f_{j}\right)^{*}\left(\Sigma^{1}\left(C_{j} \mid \Delta_{C_{j}}\right)\right)$.

\section{Quotients résolubles des groupes de Kähler or- bifoldes.}

Nous suivrons, en les adaptant au contexte orbifolde, les arguments de [De 06]. Le résultat principal est le suivant :

Théorème 4.1 Soit $(X \mid \Delta)$ une orbifolde géométrique lisse, avec $X$ Kähler compacte et connexe. On a équivalence entre les propriétés suivantes :

1. Tout quotient résoluble $R$ de $\pi_{1}(X \mid \Delta)$ est virtuellement nilpotent (ie : admet un sous-groupe d'indice fini nilpotent).

2. Il n'existe pas de morphisme orbifolde divisible $f:(X \mid \Delta) \rightarrow\left(C \mid \Delta_{C}\right)$ sur une courbe orbifolde hyperbolique.

3. Il n'existe pas de morphisme de groupes surjectif : $\pi_{1}(X \mid \Delta) \rightarrow \pi_{1}(C \mid \Delta)$, avec $(C \mid \Delta)$ une courbe orbifolde hyperbolique.

Les implications $1 \Longrightarrow 3 \Longrightarrow 2$ sont évidentes. L'implication $2 \Longrightarrow 1$ résulte des trois énoncés $4.2,4.3$ et 4.4 suivants :

Théorème 4.2 ([De 06, théorème 3.2]) Soit $R$ un groupe résoluble de type fini non virtuellement nilpotent. Il existe alors un sous-groupe $R^{\prime}$ d'indice fini de $R$ ayant un quotient métabélien $Q^{\prime}$ non virtuellement nilpotent. (Un groupe $Q^{\prime}$ est métabélien si son groupe dérivé est abélien, ie : si $\left.D(D G):=D^{2} G=\{1\}\right)$.

Théorème 4.3 Soit $(X \mid \Delta)$ une orbifolde géométrique lisse, avec $X$ kähler compacte et connexe. Si le groupe dérivé $D G^{\prime}$ de tout sous-groupe d'indice fini $G^{\prime}<$ $G:=\pi_{1}(X \mid \Delta)$ est de type fini, tout quotient résoluble de $\pi_{1}(X \mid \Delta)$ est virtuellement nilpotent.

Démonstration (de 4.3) : Il nous suffit, d'après 4.2, de montrer que $G / D^{2} G$ est virtuellement nilpotent (avec $G:=\pi_{1}(X \mid \Delta)$ ), c'est-à-dire que $G$ agit par conjugaison de manière quasi-unipotente sur $D G / D^{2} G$. Mais les valeurs propres de l'action de $G \operatorname{sur}\left(D G / D^{2} G\right) \otimes \mathbb{C}$ ne sont autres que les éléments de $\Sigma^{1}(X \mid \Delta)$, et sont donc en nombre fini, et de torsion, d'après 3.1 
Théorème 4.4 Soit $(X \mid \Delta)$ une orbifolde géométrique lisse, avec $X$ Kähler compacte et connexe. On a équivalence entre les propriétés suivantes :

1. Il existe $G^{\prime}<\pi_{1}(X \mid \Delta):=G$ d'indice fini tel que le groupe dérivé $D G^{\prime} \mathbf{n}$ 'est pas de type fini.

2. Il existe un morphisme orbifolde divisible $f:(X \mid \Delta) \rightarrow\left(C \mid \Delta_{C}\right)$ sur une courbe orbifolde hyperbolique.

Remarque 4.5 La démonstration montre plus précisément que $D G$ n'est pas de type fini si et seulement s'il existe $f:(X \mid \Delta) \rightarrow\left(C \mid \Delta_{C}\right)$ sur une courbe orbifolde hyperbolique de genre $g>0$.

Démonstration : L'implication $2 \Longrightarrow 1$ est claire, puisque $D \pi_{1}\left(C \mid \Delta_{C}\right)$ n'est pas de type fini si $\left(C \mid \Delta_{C}\right)$ est hyperbolique et de genre $g>0$. Lorsque $g=0$, un revêtement fini de $\left(C \mid \Delta_{C}\right)$ est de genre strictement positif.

L'implication opposée est, pour l'essentiel, une adaptation au cas orbifolde de celle de [De 06, théorème 1.1], dont nous adopterons les notations. Le lemme 4.6 cidessous nous permettra de supposer que $D \pi_{1}(X \mid \Delta)$ n'est pas de type fini. Il existe donc alors (dans la terminologie de [De 06]) une classe "exceptionnelle" réelle (ie : à valeurs réelles) $\chi \in \widehat{G}$, avec $G:=\pi_{1}(X \mid \Delta)$. Cette "exceptionalité" signifie, par définition (voir[BS]), que $\chi^{-1}([0,+\infty[)$ n'est pas connexe (ie : dans le graphe de Cayley $C(G)$ de $G$, le sous-graphe dont les sommets sont dans $\chi^{-1}([0,+\infty[)$, et dont les arêtes sont toutes les arêtes joignant de tels sommets n'est pas connexe, ceci pour un système générateur fini quelconque de $G$; la condition étant indépendante de ce système). L'existence d'un caractère "exceptionnel" lorsque $D G$ n'est pas de type fini est [BS, theorem 4.2], l'un des résultats de base de la théorie.

On déduit de $\chi$ une fonction $h: X_{a b} \rightarrow \mathbb{R}$ sur le revêtement abélien (sans torsion) maximal de $X$, de groupe $\pi_{1}(X)_{a b} /$ Torsion. La dérivée de cette fonction descend en une 1-forme réelle $d$-fermée $w$, partie réelle d'une 1-forme holomorphe $\omega$ sur $X$.

Soit alors $r:(\widetilde{X \mid \Delta}) \rightarrow X$ le revêtement universel de $(X \mid \Delta)$. La fonction $h$ se relève en une fonction $\widetilde{h}:(\widetilde{X \mid \Delta}) \rightarrow \mathbb{R}$ telle que $\widetilde{h}^{-1}([s,+\infty[)$ ne soit pas connexe pour $s>0$ assez grand (par le lemme 2.2 de [De 06], adapté). Plus précisément, on peut exprimer topologiquement $X-\operatorname{Supp}(\Delta)$ comme un $C W$-complexe dont le 1 -squelette est un bouquet de cercles (ne rencontrant donc pas $\operatorname{Supp}(\Delta)$ ). Son image réciproque dans $(\widetilde{X \mid \Delta})$ est alors topologiquement le graphe de Cayley de $G$ (pour le système non minimal de générateurs défini par ce bouquet de cercles).

Alors $\widetilde{h}^{-1}([s,+\infty[)$ n'est pas connexe, pour $s>0$ assez grand, puisque si $D \subset$ $(\widetilde{X \mid \Delta})$ est un domaine fondamental compact pour $G, \widetilde{h}$ est bornée sur $D$, et que $\widetilde{h}(g \cdot D)=\log (\chi(g))+\widetilde{h}(D)$.

Soit alors $\tilde{d}: \tilde{Y} \rightarrow(\widetilde{X \mid \Delta})$ une désingularisation $G$-équivariante, et $\widetilde{w}$ le relèvement à $\tilde{Y}$ de $w$. Le second argument (section 2.2.2 de [De 06], qui en est le point crucial) s'applique encore directement à notre situation, et montre que le feuilletage de $\widetilde{Y^{\prime}}$ défini par la 1-forme holomorphe $r^{*}(\omega)=d \widetilde{F}$ a une feuille $\widetilde{F}^{-1}(\psi)$ dont une composante connexe $\widetilde{C_{\omega}}$ est critique, c'est-à-dire telle que $d \widetilde{F}=0$ sur cette composante (de codimension un). L'image dans $X$ de cette composante est 
une feuille compacte du feuilletage de $X$ défini par $\omega$ puisque les fibres de $\widetilde{d}$ sont envoyées sur des sous-ensembles analytiques de codimension complexe au moins 2 , et que $r$ est finie au voisinage de chacun des points de $\widetilde{(X \mid \Delta)}$. (Remarquer que cette feuille compacte ne provient évidemment pas, en général, d'une feuille critique de l'image réciproque de $\omega$ sur le revêtement universel de $X$. L'annulation additionnelle provient de la ramification de $r$ le long des composantes de $\Delta$ ).

Il résulte alors de [DG 05,4.1] ou de [Si 93-2] que les feuilles de $\omega$ sont toutes compactes et définissent une fibration $f: X \rightarrow C$ sur une courbe de genre $g \geq 1$ telle que $\omega$ provienne de $C$. La base orbifolde $\left(C \mid \Delta_{f, \Delta}\right)$ est alors nécessairement hyperbolique, puisque l'on a une suite exacte de groupes ([Ca 07, Prop.11.7]) :

$$
1 \rightarrow \pi_{1}\left(F \mid \Delta_{F}\right) \rightarrow G \rightarrow \pi_{1}\left(C \mid \Delta_{f, \Delta}\right) \rightarrow 1,
$$

dans laquelle $\left(F \mid \Delta_{F}\right)$ est la fibre orbifolde (générique) de $f:(X \mid \Delta) \rightarrow C$, de sorte que $\pi_{1}\left(F \mid \Delta_{F}\right)$ est de type fini, la classe exceptionnelle $\chi$ provenant de $C$. En effet si $\left(C \mid \Delta_{C}\right)$ n'est pas hyperbolique, $C$ est elliptique et $\Delta_{C}=0$. On a donc une suite exacte (déduite de la précédente) :

$$
1 \rightarrow \pi_{1}\left(F \mid \Delta_{F}\right) \rightarrow \operatorname{Ker}\left(\chi \circ f_{*}\right) \rightarrow \operatorname{Ker}(\chi) \rightarrow 1
$$

Mais $\operatorname{Ker}(\chi) \subset \mathbb{Z}^{\oplus 2}$ est de type fini, donc aussi $\operatorname{Ker}(\chi)$, puisque $\pi_{1}\left(F \mid \Delta_{F}\right)$ est de type fini. Ceci contredit le fait qu'il existe un $\chi$ exceptionnel provenant de $C$ ([B-S, theorem 4.2]).

Il nous reste à réduire le cas général $D G^{\prime}$ non de type fini, pour un $G^{\prime}<$ $D \pi_{1}(X \mid \Delta)$ d'indice fini au cas où $G^{\prime}=D \pi_{1}(X \mid \Delta)$. Ceci résulte du lemme 4.6 cidessous, en appliquant ce qui précède à une classe "exceptionnelle" de $G^{\prime}$ pour obtenir la fibration $f^{\prime}$ sur le revêtement orbifolde-étale de $(X \mid \Delta)$ défini par $G^{\prime}$ (ceci bien que $X^{\prime}$ ne soit pas lisse en général)

Lemme 4.6 Soit $u:\left(X^{\prime} \mid \Delta^{\prime}\right) \rightarrow(X \mid \Delta)$ un revêtement étale fini. Si $\left(X^{\prime} \mid \Delta^{\prime}\right)$ admet une fibration sur une courbe orbifolde hyperbolique, alors $(X \mid \Delta)$ aussi.

Démonstration : On peut supposer le revêtement $u$ Galoisien de groupe $U$. Puisque la famille des fibrations de $\left(X^{\prime} \mid \Delta^{\prime}\right)$ sur une courbe orbifolde hyperbolique est finie, on peut supposer, quitte à remplacer $\left(X^{\prime} \mid \Delta^{\prime}\right)$ par un nouveau revêtement étale, que la fibration $f^{\prime}:\left(X^{\prime} \mid \Delta^{\prime}\right) \rightarrow\left(C^{\prime} \mid \Delta_{C^{\prime}}\right)$ est préservée par l'action du groupe $U$. On définit alors $f:(X \mid \Delta) \rightarrow\left(C \mid \Delta_{C}\right)$ comme quotient de $f$ par l'action de $U$. Il résulte du lemme 2.1 que $\left(C \mid \Delta_{C}\right)$ est hyperbolique

Corollaire 4.7 Soit $(X \mid \Delta)$ une orbifolde lisse et Kähler. Si $(X \mid \Delta)$ n'admet pas de morphisme orbifolde sur une courbe orbifolde hyperbolique, alors tout quotient résoluble de $\pi_{1}(X \mid \Delta)$ est virtuellement nilpotent. 


\section{Morphisme d'Albanese.}

\subsection{Factorisation de Stein du morphisme d'Albanese.}

Soit $X$ compacte Kähler et connexe. Soit $\alpha_{X}: X \rightarrow A l b(X)$ son morphisme d'Albanese, $Z$ un modèle lisse de $\alpha_{X}(A) \subset A l b(X)$, et enfin $Y$ un modèle lisse de la factorisation de Stein de $\alpha_{X}: X \rightarrow Z$ (que l'on supposera holomorphe, ainsi que $f: X \rightarrow Y$, quitte à modifier $X$, ce qui ne change pas $\left.\pi_{1}(X)\right)$.

On obtient ainsi deux applications holomorphes : $f: X \rightarrow Y$ et $g: Y \rightarrow Z$, avec $g \circ f: X \rightarrow Z$ biméromorphe à $\alpha_{X}: X \rightarrow \alpha_{X}(X)$, avec $f$ à fibres connexes et $g$ génériquement finie, telles que $\operatorname{Alb}(Z) \cong A l b(X)$.

Soit maintenant $(X \mid \Delta)$ une structure orbifolde lisse sur $X$. Elle induit sur $Y$ et $Z$ des structures orbifoldes $\Delta_{Y}:=\Delta_{f, \Delta}$ et $\Delta_{Z}:=\Delta_{g \circ f, \Delta}$. On supposera (par modification adéquate de $X, Y, Z)$ que les morphismes $f$ et $g \circ f$ sont nets au sens de [Ca 07]. Ceci implique ([Ca 07]) que pour tout morphisme $k: Z \rightarrow B$ sur une courbe projective lisse $B$, si $\Delta_{C}:=\Delta_{k, \Delta_{Z}}$, alors : $\Delta_{k \circ g \circ f, \Delta}:=\Delta_{k, \Delta_{Z}}$ (et, de manière similaire : $\left.\Delta_{k \circ g \circ f, \Delta}:=\Delta_{k \circ g, \Delta_{Y}}\right)$.

Soit alors $f_{j}:(X \mid \Delta) \rightarrow\left(C_{j} \mid \Delta_{j}\right)$ l'ensemble (fini) des morphismes orbifoldes surjectifs et à fibres connexes sur des orbifoldes de courbes hyperboliques de genre $g>0$, notant pour tout $j: \Delta_{j}:=\Delta_{f_{j}, \Delta}$.

On notera enfin : $h: X \rightarrow \Pi_{j} C_{j}$ le morphisme produit des $f_{j}$, et $V \subset \Pi_{j} C_{j}$ son image désingularisée. On notera encore $h: X \rightarrow V$ le morphisme résultant (après modification de $X)$. On supposera encore que $h: X \rightarrow V$ est net. On munira enfin $V$ de la structure orbifolde $\Delta_{V}:=\Delta_{h, \Delta}$.

\subsection{Green-Lazarsfeld et morphisme d'Albanese.}

Si $(X \mid \Delta)$ est une orbifolde Kählérienne, on note $\Sigma_{c}^{1}(X \mid \Delta)$ la partie continue de $\Sigma^{1}(X \mid \Delta)$, de sorte que $\Sigma^{1}(X \mid \Delta)$ est réunion de $\Sigma_{c}^{1}(X \mid \Delta)$ et d'un nombre fini de points isolés (qui sont de torsion).

Proposition 5.1 Dans la situation et avec les notations du $§ 5.1$ précédentes, les applications naturelles déduites de $f, g, h$ induisent des bijections :

$\Sigma_{c}^{1}\left(V \mid \Delta_{V}\right) \rightarrow \Sigma_{c}^{1}\left(Z \mid \Delta_{Z}\right) \rightarrow \Sigma_{c}^{1}\left(Y \mid \Delta_{Y}\right) \rightarrow \Sigma_{c}^{1}\left(X \mid \Delta_{X}\right)$.

Démonstration : Elle se déduit par fonctorialité du fait que $\Sigma_{c}^{1}\left(X \mid \Delta_{X}\right)$ est la réunion des $f_{j}^{*}\left(\Sigma^{1}\left(C_{j} \mid \Delta_{j}\right)\right.$ (avec une égalité similaire pour les autres), et du fait que $\left.\Delta_{k \circ g \circ f, \Delta}:=\Delta_{k \circ g, \Delta_{Y}}\right)($ et des autres égalités analogues)

Ces égalités suggèrent de comparer les quotients résolubles des groupes fondamentaux des 4 orbifoldes précédentes. 


\subsection{Quotients résolubles et morphisme d'Albanese.}

Les applications $f: X \rightarrow Y, g: Y \rightarrow Z$ et $h: Z \rightarrow V$ induisent des morphismes de groupes naturels $f_{*}: G:=\pi_{1}(X \mid \Delta) \rightarrow \pi_{1}\left(Y \mid \Delta_{Y}\right):=H, g_{*}: H:=\pi_{1}\left(Y \mid \Delta_{Y}\right) \rightarrow$ $\pi_{1}\left(Z \mid \Delta_{Z}\right):=K$, et $h_{*}: K=\pi_{1}\left(Z \mid \Delta_{Z}\right) \rightarrow \pi_{1}\left(V \mid \Delta_{V}\right):=J$.

On notera aussi : $L:=\operatorname{Ker}\left(f_{*}\right), M:=\operatorname{Ker}\left((g \circ f)_{*}\right)$, et $N:=\operatorname{Ker}\left((h \circ g \circ f)_{*}\right)$. Ce sont des sous-groupes normaux de $G$. On a bien sûr des inclusions : $L<M<N$. Remarquer que $N=G$ lorsque $\Sigma_{c}^{1}(X \mid \Delta)=\emptyset \mathrm{ou}$, de manière équivalente, lorsque $(X \mid \Delta)$ n'a pas de fibration sur une courbe orbifolde hyperbolique de genre $g>0$.

Un morphisme de groupes surjectif $\varphi: G \rightarrow R$, avec $R$ résoluble sera appelé un quotient résoluble de $G$. Les images $\varphi(L), \varphi(M)$, et $\varphi(N)$ sont donc alors des sous-groupes (résolubles) normaux de $R$.

Question 5.2 Avec les notations de 5.1 ci-dessus, soit $\varphi: G \rightarrow R$ un morphisme de groupes surjectif, avec $R$ résoluble (on dira que $R$ est un quotient résoluble de $G)$.

1. Les groupes $\varphi(L)$ et $\varphi(M)$ sont-ils finis?

2. Le groupe $\varphi(N)$ est-il virtuellement nilpotent?

Une réponse affirmative à la question 5.2.1 signifierait donc que les quotients résolubles de $G$ et de $K$ sont les mêmes, à commensurabilité près. Et donc que ces quotients sont ceux des (groupes fondamentaux) orbifoldes de sous-variétés de tores complexes. Il est démontré dans [Ca 95] que c'est le cas pour les quotients nilpotents de $G$.

Nous allons répondre à ces questions dans le cas de $L$ seulement.

Théorème 5.3 Avec les notations précédentes, soit $(X \mid \Delta)$ une orbifolde lisse et Kähler, et $\varphi: G \rightarrow R$, un quotient résoluble de $G:=\pi_{1}(X \mid \Delta)$. Alors :

1. Si $(X \mid \Delta)$ n'a pas de fibration sur une courbe hyperbolique, alors le groupe $\varphi(L)$ est fini, et le groupe $\varphi(N)=R$ est virtuellement nilpotent.

Dans le cas général:

2. $\varphi(L)$ est fini si $L$ est résiduellement fini dans $G$ (voir définition 5.4 cidessous).

3. $\varphi(L)$ est fini.

Définition 5.4 Soit $L<G$ un sous-groupe normal. Nous dirons que $L$ est résiduellement fini dans $G$ si, pour tout sous-groupe $L^{\prime}<L$ d'indice fini, il existe un sous-groupe $G^{\prime}<G$ d'indice fini tel que : $(G \cap L) \subset L^{\prime}$.Par exemple :

1. Si $G$ est résiduellement fini, tout $L<G$ est résiduellement fini dans $G$.

2. Si $G=(G / L) \ltimes L$ est produit semi-direct de $L, L$ de type fini, et d'un sous-groupe $G / L$, alors $L$ est résiduellement fini dans $G$. (En effet : si $L^{\prime}$ est caractéristique dans $L$, alors $G^{\prime}:=(G / L) \ltimes L^{\prime}$ résoud le problème pour $L^{\prime}$. Or tout 
sous-groupe d'indice fini de $L$ contient un sous-groupe caractéristique de $L$ d'indice fini, puisque L est de type fini).

Remarque 5.5 La finitude de $\varphi(M)$ est plus délicate que celle de $\varphi(L)$, car non préservée a priori par revêtements orbifolde-étales.

Démonstration : Si $(X \mid \Delta)$ n'a pas de fibration sur une courbe hyperbolique, alors $N=G$, et tout quotient résoluble de $G$ est virtuellement nilpotent par 4.7, ce qui établit la seconde partie de la première assertion. La finitude $\varphi(L)$ dans ce cas est un cas particulier de l'assertion 2 démontrée ci-dessous, puisque $R=\varphi(N)$ est alors virtuellement nilpotent, donc résiduellement fini.

La preuve des secondes assertions découle directement du résultat 5.6 suivant : en effet, d'après [Ca 01, 3.6.1] appliqué à $S=\varphi(L)$, un groupe résoluble $S$ est fini si $S_{a b}^{\prime}$ est fini pour tout sous-groupe $S^{\prime}<S$ d'indice fini.

Nous démontrerons l'assertion 3 après l'énoncé 5.6

Théorème 5.6 (Voir $\left[\right.$ Ca 01, théorème 4.1]) Soit $f:(X \mid \Delta) \rightarrow\left(Y \mid \Delta_{Y}\right)$ l'application définie ci-dessus, et $\varphi: G \rightarrow R$ un quotient résoluble de $G$.

Alors, pour tout sous-groupe $G^{\prime}$ d'indice fini de $G:\left(\varphi\left(L \cap G^{\prime}\right)\right)_{a b}$ est un groupe fini.

Démonstration : Soit $\left(F \mid \Delta_{F}\right)$ l'une des fibres orbifoldes génériques de $f$ : $(X \mid \Delta) \rightarrow(X \mid \Delta)$. Alors $L \subset G$ est aussi l'image de $\pi_{1}\left(F \mid \Delta_{F}\right)$ dans $G:=\pi_{1}(X \mid \Delta)$ (par $[\mathrm{Ca} 07,11.2])$. Le résultat est donc établi lorsque $\Delta=\Delta_{Y}=0$ dans [Ca 01, 4.1]. La démonstration de loc. cit. s'adapte en fait directement au cas plus général considéré ici en remarquant que $\left(\pi_{1}\left(F \mid \Delta_{F}\right)\right)_{a b} /$ Torsion $=\left(\pi_{1}(F)\right)_{a b} /$ Torsion

Démonstration de 5.3.3 : Elle résulte immédiatemment de 5.3 et des trois lemmes 5.7, 5.8, 5.9 ci-dessous : en effet, avec les notations de 5.8, 5.3 et 5.7 montrent que l'assertion est vraie pour $\left(X^{\prime} \mid \Delta^{\prime}\right)$, et 5.9 montre que l'assertion pour $\left(X^{\prime} \mid \Delta^{\prime}\right)$ l'implique pour $(X \mid \Delta)$ aussi

Lemme 5.7 Soit $f:(X \mid \Delta) \rightarrow\left(Y \mid \Delta_{Y}\right)$, avec $\left.\Delta_{Y}:=\Delta_{f, \Delta}\right),\left(F \mid \Delta_{F}\right)$ la fibre orbifolde générique de $f$, et $f$ nette. Si $f$ admet une section orbifolde divisible $\sigma:\left(Y \mid \Delta_{Y}\right) \rightarrow$ $(X \mid \Delta)$, alors la suite exacte naturelle :

$$
\pi_{1}\left(F \mid \Delta_{F}\right) \rightarrow \pi_{1}(X \mid \Delta) \rightarrow \pi_{1}\left(Y \mid \Delta_{Y}\right) \rightarrow 1
$$

est scindée, et l'image $L$ de $\pi_{1}\left(F \mid \Delta_{F}\right)$ dans est résiduellement finie dans $G:=$ $\pi_{1}\left(X \mid \Delta_{X}\right)$.

Rappelons [Ca 07, §5] que $\sigma$ est donc une section de $f$ et un morphisme orbifolde divisible.

\footnotetext{
${ }^{7}$ Dans [Ca 01, 4.1], on affirme que $\varphi\left(L \cap G^{\prime}\right)$ est lui-même fini. La démonstration établit d'abord la finitude de l'abélianisé, et en déduit la finitude de ce groupe par l'intermédiaire du lemme [Ko 93 : J. Kollàr. Inv. Math.113 (1993), 177-215, Prop. 6.4] dont la démonstration est cependant incomplète. Nous n'énonçons donc dans 5.6 que la partie de ce résultat qui ne dépend pas de [Ko 93, 6.4], et établissons donc [Ca 01, 4.1] intégralement dans 5.3.
} 
Démonstration : Le morphisme orbifolde $\sigma$ induit [Ca 07, §11] un morphisme de groupes $\sigma_{*}: \pi_{1}\left(Y \mid \Delta_{Y}\right) \rightarrow \pi_{1}(X \mid \Delta)$ dont l'image $\Sigma$ est un supplémentaire de $L$ dans $\pi_{1}(X \mid \Delta)$, qui est donc produit semi-direct de $\Sigma$ et de $L$. Donc $L$ est résiduellement fini dans $G \bullet$

Lemme 5.8 Soit $f:(X \mid \Delta) \rightarrow\left(Y \mid \Delta_{Y}\right)$ comme ci-dessus. Il existe alors un diagramme commutatif de morphismes orbifoldes (divisibles):

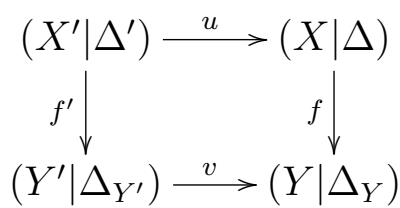

tel que :

1. Les variétés $X^{\prime}, Y^{\prime}$ sont Kähler.

2. Les fibres orbifoldes génériques de $f$ et $f^{\prime}$ coincident (ie: $f^{\prime}$ est génériquement déduite de $f$ par le changement de base $v$ ).

3. $f^{\prime}:\left(X^{\prime} \mid \Delta^{\prime}\right) \rightarrow\left(Y^{\prime} \mid \Delta_{Y^{\prime}}\right)$ admet une section orbifolde $\sigma$.

Démonstration : Soit $Y^{\prime}$ un modèle lisse Kähler de $X \times_{Y} X$. Il existe donc une section tautologique $\sigma: Y^{\prime} \rightarrow X^{\prime}:=Y^{\prime} \times_{Y} X$ de la fibration $f^{\prime}: X^{\prime} \rightarrow Y^{\prime}$. Nous noterons encore $X^{\prime}$ un modèle lisse adéquat de $Y^{\prime} \times_{Y} X$. On choisit alors sur $X^{\prime}$ une structure orbifolde géométrique $\Delta^{\prime}$ telle que :

1. La fibre orbifolde générique $\left(F^{\prime} \mid \Delta_{F^{\prime}}\right)$ soit égale à son image $\left(F \mid \Delta_{F}\right)$.

2. Le morphisme naturel $u:\left(X^{\prime} \mid \Delta^{\prime}\right) \rightarrow(X \mid \Delta)$ soit un morphisme orbifolde : c'est toujours possible en augmentant (au sens divisible) les multiplicités sur les composantes $f^{\prime}$-verticales du support de $\Delta^{\prime}$.

3. La fibration $f^{\prime}$ est nette.

On définit alors $\Delta_{Y}^{\prime}:=\Delta_{f^{\prime}, \Delta^{\prime}}$.

En général, la section $\sigma:\left(Y^{\prime} \mid \Delta_{Y^{\prime}}\right) \rightarrow\left(X^{\prime} \mid \Delta^{\prime}\right)$ n'est pas un morphisme orbifolde (la $\Delta_{Y^{\prime}}$-multiplicité d'un diviseur $E^{\prime}$ irréductible effectif de $Y^{\prime}$ peut diviser strictement celle des composantes $D^{\prime}$ de $\Delta^{\prime}$ que rencontre $\sigma$ au-dessus de $E^{\prime}$ ). On peut néammoins, pour tout tel $E^{\prime}$, augmenter au sens divisible les $\Delta^{\prime}$-multiplicités des composantes irréductibles de $\left(f^{\prime}\right)^{*}\left(E^{\prime}\right)$ de telle sorte que la $\Delta_{Y^{\prime}}$-multiplicité de $E^{\prime}$ soit égale à la plus grande des $\Delta^{\prime}$-multiplicités des composantes $D^{\prime}$. La section $\sigma:\left(Y^{\prime} \mid \Delta_{Y^{\prime}}\right) \rightarrow\left(X^{\prime} \mid \Delta^{\prime}\right)$ est alors un morphisme orbifolde divisible

Lemme 5.9 Dans la situation de 5.8, les images de $F^{\prime}$ dans $A l b\left(X^{\prime}\right)$ et de $F$ dans $A l b(X)$ ont la même dimension.

Plus précisément, $A l b\left(X^{\prime}\right)=\operatorname{Alb}(X) \times_{A l b(Y)} A l b\left(Y^{\prime}\right)$.

Démonstration : Ceci résulte de la commutativité du diagramme : 


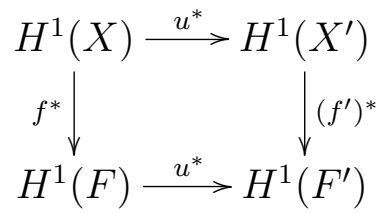

dans lequel les coefficients sont rationnels

\section{Orbifoldes spéciales.} ici.

Nous renvoyons à [Ca 04] et [Ca 07] pour plus de détails sur les notions utilisées

Soit $(X \mid \Delta)$ une orbifolde lisse et Kähler. Rappelons ([Ca 07, 8.1]) que $(X \mid \Delta)$ est dite spéciale s'il n'existe pas de fibration méromorphe (au sens orbifolde) dominante $g:(X \mid \Delta) \rightarrow\left(Y \mid \Delta_{Y}\right)$ sur une orbifolde $\left(Y \mid \Delta_{Y}\right)$ de type général avec $\operatorname{dim}(Y)=$ $\kappa\left(Y \mid \Delta_{Y}\right)>0$. Il existe d'autres définitions équivalentes.

- Les exemples fondamentaux de telles orbifoldes sont les $(X \mid \Delta)$ qui sont soit rationnellement connexes, soit avec $\kappa(X \mid \Delta)=0$. Une fibration dont la fibre orbifolde générique et la base orbifolde sont spéciales est spéciale (une propriété fausse dans la catégorie des variétés sans structure orbifolde). Enfin, si $a_{X}: X \rightarrow W$ est la réduction algébrique de $X$, et $(X \mid \Delta)$ une structure orbifolde sur $X$, la fibre orbifolde générique $\left(F \mid \Delta_{F}\right)$ de $a_{X}$ est spéciale (ceci est démontré dans [Ca 04] lorsque $\Delta=0$, mais la démonstration s'adapte sans changement au cas général).

La conjecture centrale concernant le groupe fondamental de ces orbifoldes géométriques est (voir [Ca 07]) :

Conjecture 6.1 Si $(X \mid \Delta)$ est spéciale, alors $\pi_{1}(X \mid \Delta)$ est virtuellement abélien.

Nous allons vérifier cette conjecture pour les quotients résolubles de $\pi_{1}(X \mid \Delta)$, et pour une classe un peu plus large d'orbifoldes.

Théorème 6.2 Soit $(X \mid \Delta)$ une orbifolde lisse et Kähler. Si, pour tout revêtement orbifolde-étale fini $r:\left(X^{\prime} \mid \Delta^{\prime}\right) \rightarrow(X \mid \Delta)$, l'application d'Albanese $\alpha_{X^{\prime}}: X^{\prime} \rightarrow$ $A l b\left(X^{\prime}\right)$ est surjective ${ }^{8}$, alors tout quotient virtuellement résoluble de $\pi_{1}(X \mid \Delta)$ est virtuellement abélien.

Démonstration : Hypothèse et conclusion sont préservées par revêtements orbifolde-étale. L'hypothèse implique évidemment que $(X \mid \Delta)$ n'a pas de fibration sur

\footnotetext{
${ }^{8}$ Une condition plus faible (également nécessaire) est en fait suffisante : l'injectivité de l'application $\left(\alpha_{X^{\prime}}\right)^{*}: H^{2}\left(A l b\left(X^{\prime}\right), \mathbb{Q}\right) \rightarrow H^{2}\left(X^{\prime}, \mathbb{Q}\right)$. Voir $[$ Ca 95$]$ : la démonstration est la même.
} 
une courbe orbifolde hyperbolique. Les quotients résolubles de $\pi_{1}(X \mid \Delta)$ sont donc virtuellement nilpotents. Il suffit donc (après revêtement orbifolde-étale adéquat) de montrer que si un tel quotient $R$ est nilpotent et sans torsion, il est virtuellement abélien. Mais alors $R$ est aussi un quotient de $\pi_{1}(X)$, et le résultat principal de [Ca 95] implique l'assertion

Corollaire 6.3 Soit $(X \mid \Delta)$ une orbifolde lisse et Kähler spéciale, et $R$ un groupe quotient de $\pi_{1}(X \mid \Delta)$. Alors $R$ est virtuellement abélien dans les deux cas suivants:

1. $R$ est virtuellement résoluble.

2. $R<G l(n, \mathbb{C})$ est linéaire.

De plus, si $q\left(X^{\prime}\right)=0$ pour tout revêtement étale fini $X^{\prime}$ de $X$, alors $R$ est fini.

Démonstration : Si $R$ est virtuellement résoluble, il suffit, par 6.2 de vérifier que l'application d'Albanese de $X$ est surjective, puisque les revêtements orbifolde-étales finis de $(X \mid \Delta)$ sont spéciaux. Mais $X$ étant elle-même spéciale, cette surjectivité résulte de [Ca 04]. Si $R$ est linéaire, il admet, par le lemme de Selberg, un sousgroupe $R^{\prime}$ d'indice fini sans torsion, qui est donc un quotient de $\pi_{1}(X)$ (puisque le noyau de $\pi_{1}(X \mid \Delta) \rightarrow \pi_{1}(X)$ est engendré par des éléments de torsion). Puisque $X$ est aussi spéciale, l'assertion résulte encore de [Ca 04].

Pour démontrer la dernière assertion, supposons (après revêtement orbifoldeétale fini adéquat) $R$ abélien infini sans torsion. Alors $\left(\pi_{1}(X \mid \Delta)\right)_{a b}=\left(\pi_{1}(X)\right)_{a b}$ est infini. Contradiction puisque $q\left(X^{\prime}\right)=0$ par hypothèse

Nous utiliserons dans le $\S 7$ le cas particulier suivant :

Corollaire 6.4 Soit $(X \mid \Delta)$ une orbifolde lisse et Kähler, avec $X$ de dimension algébrique nulle (ie : $a(X)=0$, ou encore: les fonctions méromorphes globales sur $X$ sont constantes). Soit $R$ un groupe quotient de $\pi_{1}(X \mid \Delta)$. Alors $R$ est virtuellement abélien dans les deux cas suivants :

1. $R$ est virtuellement résoluble.

2. $R<G l(n, \mathbb{C})$ est linéaire.

De plus, si $q\left(X^{\prime}\right)=0$ pour tout revêtement étale fini $X^{\prime}$ de $X$, alors $R$ est fini.

\section{Réduction algébrique.}

On montre ici que les ensembles de Green-Lazarsfeld et les quotients résolubles du groupe fondamental d'une orbifolde Kähler coincident essentiellement (à un facteur abélien fixe près) avec ceux de sa réduction algébrique, ce qui permet de se ramener au cas projectif. Nous renvoyons à [Ca 01] et [U 75] pour les notions relatives à la réduction algébrique.

Théorème 7.1 (Voir [Ca 01, 3.1]) Soit $a_{X}: X \rightarrow W$ une réduction algébrique, avec $X$ compacte Kähler.

On note $G:=\pi_{1}(X \mid \Delta)$, et $Q:=\pi_{1}\left(W \mid \Delta_{W}\right)$, avec $\Delta_{W}:=\Delta_{a_{X}, \Delta}$. 
Soit $\chi \in \Sigma_{c}^{1}(G)$, et $u \in \operatorname{Hom}_{G}\left(D G, \mathbb{C}_{\chi}\right)$. Il existe alors $\chi^{\prime} \in \Sigma_{c}^{1}(Y)$ tel que : $\chi=\left(a_{X}\right)^{*}\left(\chi^{\prime}\right)$, et $v \in \operatorname{Hom}_{Q}\left(D Q, \mathbb{C}_{\chi^{\prime}}\right)$ tel que $: u=\left(a_{X}\right)^{*}(v)$.

Démonstration : L'assertion résulte de 5.1

- L'étude des quotients résolubles de $G:=\pi_{1}(X \mid \Delta)$ est basée sur le lemme 7.2 ci-dessous. Considérons le diagramme commutatif :

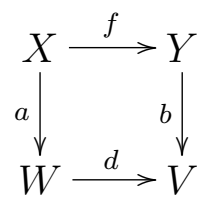

dans lequel $f$ (resp. $d$ ) est la factorisation de Stein du morphisme d'Albanese de $X$ (resp. $W$ ) introduit au $\S 5.1$ ci-dessus, tandis que $a$ est la réduction algébrique de $X$, et $b$ déduite des morphismes d'Albanese de $X$ et $W$.

Lemme 7.2 Dans le diagramme précédent, on a les propriétés suivantes:

1. $b$ est la réduction algébrique de $Y$.

2. Si $D \subset Y$ est un diviseur effectif irréductible quelconque, alors $b(D) \subsetneq V$.

3. Le morphisme naturel : $Y \rightarrow V \times_{A l b(W)} A l b(X)$ est biméromorphe (et surjectif). 4. $b: Y \rightarrow V$ est un fibré pincipal de fibre $B:=\operatorname{Ker}(A l b(X) \rightarrow A l b(V))$.

Démonstration : Assertion 1. Nous utiliserons les deux faits suivants ([Ca 85] ou [F 83]) :

1. Pour tout $X$, l'application naturelle $X \rightarrow W \times_{A l b(W)} A l b(X)$ est surjective.

2. Il n'existe pas de diviseur effectif de $W \times_{A l b(W)} A l b(X)$ surjectif sur $W$ (d' où résulte l'assertion2).

La connexité des fibres de $b$ résulte de celle des fibres de $a$ et $d$. Si $b$ n'etait pas la réduction algébrique de $Y$, il existerait des fibrations $Y \rightarrow V_{1}$ et $V_{1} \rightarrow V$ avec $V_{1}$ projectif et $\operatorname{dim}(V 1)>\operatorname{dim}(V)$. On aurait donc par définition de la réduction algébrique de $X$ une factorisation $W \rightarrow V_{1}$ de $X \rightarrow V_{1}$. Passant aux morhismes d'Albanese, on aurait des morphismes $W \rightarrow \operatorname{Alb}\left(V_{1}\right) \rightarrow \operatorname{Alb}(V)$, le morphisme $A l b\left(V_{1}\right) \rightarrow A l b(V)$ étant surjectif de noyau de dimension positive (puisque $V \rightarrow A l b(V)$ est génériquement finie sur son image, et la surjectivité de $\left.Y \rightarrow V_{1} \times_{A l b\left(V_{1}\right)} A l b(Y)\right)$. Ceci contredit le fait que $V$ est l'image de la factorisation de Stein du morphisme d'Albanese de $W$.

Assertion 3. La surjectivité provient du rappel 1. ci-dessus. Le fait 2. montre que le morphisme $Y \rightarrow V \times \times_{A l b(W)} A l b(X)$ ne ramifie pas au-dessus de la fibre générique de $V \times_{A l b(W)} A l b(X) \rightarrow V$. Donc $Y_{v} \rightarrow B$ est étale pour $v \in V$ générique, et $Y_{v}$ est donc un tore complexe isogène à $B:=\operatorname{Ker}(A l b(X) \rightarrow A l b(W))$. Les assertions 3. et 4. résulteront alors du fait que ce morphisme $Y_{v} \rightarrow B$ est un isomorphisme. Si ce n'était pas le cas, $\left.\operatorname{Ker}\left(b_{*}:\left(\pi_{1}(Y)\right)_{a b} \rightarrow \pi_{1}(V)\right)_{a b}\right) /$ Tor sion serait un sous-groupe 
strict d'indice fini de $\pi_{1}(B)=\operatorname{Ker}\left(b_{*}: \pi_{1}(A l b(Y)) \rightarrow \pi_{1}(\operatorname{Alb}(V))=\operatorname{Ker}\left(b_{*}\right.\right.$ : $\left.\left.\left(\pi_{1}(Y)\right)_{a b} \rightarrow \pi_{1}(V)\right)_{a b}\right) /$ Torsion, une contradiction

- Soit maintenant $\Delta$ une structure orbifolde géométrique lisse quelconque sur $X$.

Soit $\Delta_{W}:=\Delta_{a, \Delta}, \Delta_{Y}:=\Delta_{f, \Delta}, \Delta_{V}:=\Delta_{b \circ f, \Delta}$ les structures orbifoldes induites sur $W, Y$, et $V$ respectivement. Puisque l'on peut supposer $f$ et $a$ "nettes", on a donc aussi : $\Delta_{b \circ f, \Delta}=\Delta_{b, \Delta_{f, \Delta}}$, par [Ca 07, 3.14].

On notera aussi (comme ci-dessus) $: H:=\pi_{1}\left(Y \mid \Delta_{Y}\right), Q:=\pi_{1}\left(W \mid \Delta_{W}\right)$, et $T:=$ $\pi_{1}\left(V \mid \Delta_{V}\right)$.

Nous avons donc un diagramme comutatif de morphismes de groupes à lignes et colonnes exactes :

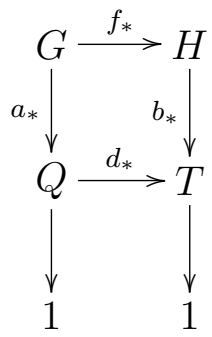

Corollaire 7.3 Avec ces notations, on a une suite exacte naturelle induite par $b$ :

$$
1 \rightarrow \pi_{1}(B) \rightarrow H \rightarrow T \rightarrow 1
$$

Cette suite est (non canoniquement) scindée par des scindages induisant des produits de groupes: $H \cong \pi_{1}(B) \times T$.

Démonstration : On a (par [Ca 07, 11.2]) une suite exacte de groupes :

$$
1 \rightarrow \pi_{1}\left(B \mid \Delta_{B}\right) \rightarrow H \rightarrow T \rightarrow 1,
$$

où $\left(B \mid \Delta_{B}\right)$ est la restriction de $\Delta_{Y}$ à une fibre générique $F \cong B$ de $b$. Mais il n'existe pas (par 7.2.2 ci-dessus) de diviseur effectif $D$ sur $Y$ rencontrant $B$. Donc $\Delta_{B}=0$.

On a donc un diagramme commutatif à lignes et colonnes exactes :

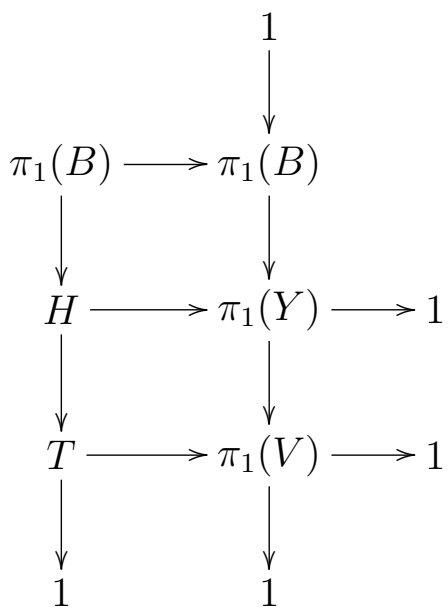


d'où l'on déduit aisément l'assertion, en scindant la suite exacte

$$
1 \rightarrow \pi_{1}(B) \rightarrow H \rightarrow T \rightarrow 1
$$

à l'aide de l'image inverse dans $H$ d'un groupe $\widetilde{\pi_{1}(V)}<\pi_{1}(Y)$ isomorphe à $\pi_{1}(V)$ et induisant un isomorphisme : $\pi_{1}(Y) \cong \pi_{1}(V) \times \pi_{1}(B)$

Le résultat suivant découle alors immédiatemment de 7.3 :

Théorème 7.4 Soit $\varphi: G:=\pi_{1}(X \mid \Delta) \rightarrow R$ un quotient résoluble de $G$. Soit (comme ci-dessus) $L:=\operatorname{Ker}\left(f_{*}: G \rightarrow H\right)$. Alors :

1. $\varphi(L)$ est un sous-groupe fini normal de $R$ (par 5.3). Soit $\rho: R \rightarrow R^{\prime}:=$ $R / \varphi(L)$ le quotient, et $\varphi^{\prime}:=\rho \circ \varphi: G \rightarrow R^{\prime}$.

2. Soit $A:=\operatorname{Ker}\left(a_{*}: G \rightarrow Q\right)$. Alors : $\varphi^{\prime}(A)$ est un sous-groupe abélien central de $R^{\prime}$, isomorphe à un quotient de $\pi_{1}(B):=\operatorname{Ker}\left(a_{*}: G_{a b} \rightarrow Q_{a b}\right) /$ Tor sion.

3. Pour tout entier $k \geq 0$ :

$$
\left(G / D^{k+1} G\right) \equiv\left(H / D^{k+1} H\right) \cong\left(T / D^{k+1} T\right) \times \pi_{1}(B) \equiv\left(Q / D^{k+1} Q\right) \times \pi_{1}(B),
$$

où : $A \equiv C$ signifie que les groupes $A$ et $C$ ont des quotients isomorphes par des sous-groupes d'indice fini.

Autrement dit : les quotients résolubles cannoniques de $G$ sont (à un quotient d'un groupe fini fixe près) des produits de ceux de $Q$ (groupe fondamental de la réduction algébrique de $(X \mid \Delta))$ par des quotients de $\pi_{1}(B)$.

Remarque 7.5 Une réponse positive à la question 5.2.1 pour le groupe $\varphi(M)$ permettrait, à l'aide des arguments de remplacer $Y$ et $V$ par les images d'Albanese de $X$ et $W$ dans les énoncés précédents. Pour les quotients nilpotents, ces énoncés concernant les images d'Albanese de $X$ et $W$ sont vrais, par [Ca 95].

\section{Quotients nilpotents des groupes de Kähler.}

\subsection{Rappels sur quotients et groupes nilpotents.}

Soit $G$ un groupe, et $s \geq 1$ un entier. On note $C^{s}(G)$ le $s$-ième terme de sa suite centrale descendante, définie par récurrence par : $C^{1}(G):=G$, et $C^{s+1}(G):=$ $\left[G, C^{s}(G)\right]$, le groupe engendré par les commutateurs de $G$ et de $C^{s}(G)$ si $s>1$. Pour tout $s \geq 1, C^{s}(G)$ est un sous-groupe caractéristique de $G$.

Le groupe $G$ est dit nilpotent de classe au plus $s$ si $C^{s+1}(G)$ est trivial (ie : réduit à son élément neutre). On note alors $\nu(G)$ le plus petit entier $s \geq 1$ tel que $C^{s+1}(G)=\{1\}$. On appelle $\nu(G)$ la classe de nilpotence (exacte) de $G$. Donc $\nu(G)=1$ (resp 2) si et seulement si $G$ est trivial (resp. abélien non trivial).

Une extension $1 \rightarrow Z \rightarrow G \rightarrow H \rightarrow 1$ d'un groupe nilpotent $H$ par un groupe abélien $Z$ est nilpotente si elle est centrale (ie : si $Z \subset Z(G)$, le centre de $G$ ). 
On notera $q_{s}: G \rightarrow G / C^{s+1}(G):=G_{s}$ le quotient naturel : $G_{s}$ est ainsi le plus grand quotient nilpotent de $G$ de classe au plus $s$ (les autres étant des quotients de $\left.G_{s}\right)$. En général, $\nu\left(G_{s}^{*}\right)<s$ (par exemple si $G$ abélien, $\nu\left(G_{s}^{*}\right)=1$ pour tout $s>1$ ).

La plupart des propriétés de finitude des groupes abéliens s'étendent aux groupes nilpotents (mais non pas aux groupes résolubles). Si $G$ est un groupe nilpotent, alors (voir [Ro] pour les 4 premières propriétés, et $[R]$ pour la dernière) :

1. L'ensemble des éléments de torsion $T(G)$ est un sous-groupe (caractéristique) de $G$. On note $\tau_{G}: G \rightarrow G^{*}:=G / T(G)$ le quotient. Alors $G^{*}$ est sans torsion. On notera $\nu^{*}(G):=\nu\left(G^{*}\right) \leq \nu(G)$.

2. Si $G$ est de type fini, $T(G)$ est fini.

3. Si $G$ est de type fini, il est de présentation finie.

4. Si $G$ est de type fini, il est linéaire (ie : plongeable dans un $G l(n, \mathbb{R})$ ). Plus précisément :

5. Si $G$ est nilpotent, sans torsion, et de type fini, il existe un unique groupe de Lie réel et simplement connexe $G_{\mathbb{R}}$, défini sur $\mathbb{Q}$, tel que $G$ soit un réseau cocompact de $G_{\mathbb{R}}$. (On peut définir $G_{\mathbb{Q}}$ intrinsèquement comme la réunion des ensembles des racines $m$-ièmes (uniques, car $G$ est nilpotent sans torsion) des éléments de $G$, pour $m>0$ entier). Le groupe $G_{\mathbb{R}}$ est la complétion de Malçev de $G$. On notera alors $L G$ son algèbre de Lie (définie sur $\mathbb{Q}$ ). On a donc aussi, pour tout $s>0$ : $L\left(C^{s}(G)\right)=C^{s} L(G)$, en notant $C^{L}$ le $s$-ième terme de la suite descendante d'une algèbre de Lie $G$. Si $G$ est un groupe de type fini, $G_{s}^{*}$ est aussi de type fini. On notera donc $L C^{s}\left(G_{s}^{*}\right):=L C^{s}(G)$. Remarquer que $L C^{s}\left(G_{m+s}^{*}\right)=L C^{s}\left(G_{s}^{*}\right)$, pour tout entier $m \geq 0$.

D'après la propriété 1 ci-dessus, si $G$ est un groupe arbitraire, et $s>0$ entier, on peut donc définir la composée $q_{s}^{*}:=\left(\tau_{G_{s}}\right) \circ q_{s}: G \rightarrow G_{s}^{*}$, et $C_{*}^{s+1}(G):=\operatorname{Ker}\left(q_{s}^{*}\right)$, que nous appellerons (abusivement) $(s+1)$-ième terme de la suite entrale descandante sans torsion de $G$.

Les constructions précédentes sont évidemment fonctorielles en $G$. Si $f: G \rightarrow H$ est un morphisme de groupes, il induit donc des morphismes $f_{s}: C^{s}(G) \rightarrow C^{s}(H)$, $f^{s}: G_{s} \rightarrow H_{s}, f_{s}^{*}: G_{s}^{*} \rightarrow H_{s}^{*}$, surjectifs si $f$ l'est. Et aussi, si $G, H$ sont de type fini et sans torsion : $f_{\mathbb{R}}: G_{\mathbb{R}} \rightarrow H_{\mathbb{R}}$, ainsi que $L f: L G \rightarrow L H$, qui peuvent être composés avec les précédents.

\subsection{Morphismes stricts de groupes}

Définition 8.1 Soit $f: G \rightarrow H$ un morphisme de groupes, $G$ et $H$ étant de type fini. On dit que $f$ est un morphisme strict si, pour tout entier $s \geq 1$, le morphisme induit $L f_{s}^{*}: L C^{s}(G) \rightarrow L C^{s}(H)$ est strict, c'est-à-dire, si : $f\left(L C^{s}(G)\right) \cap L C^{j}(H)=$ $f\left(L C^{j}(G)\right.$ ), pour tout $0 \leq j \leq s$. (En général, le second membre est seulement inclus dans le premier).

Si $G=K \times H$ est le produit direct de deux groupes, et si $f$ est la seconde projection, alors $f$ est un morphisme strict. Cette notion ne semble pas avoir de caractérisation ou de propriétés de stabilité de formulation simple en termes 
algébriques. Par contre, la géométrie Kählérienne en fournit de nombreux exemples (qui inspirent la terminologie) :

Théorème 8.2 Soit $g: X \rightarrow Y$ une application holomorphe entre variétés Kählériennes compactes et connexes, et $f=g_{*}: \pi_{1}(X) \rightarrow \pi_{1}(Y)$ le morphisme induit (on omet les points-base). Alors $f$ est un morphisme de groupes strict.

Démonstration : C'est une conséquence directe de deux résultats profonds. Le premier, dû à R. Hain ( voir [H 87], et [P-S, chap. 8]), affirme que, pour tout entier $s>0$, l'algèbre de Lie $L C^{s}\left(\pi_{1}(X)\right)$ est munie d'une structure de Hodge mixte naturelle, fonctorielle en $X$, avec pour filtration par le poids : $W^{j}\left(L C^{s}\left(\pi_{1}(X)\right)\right)=$ $L C^{j}\left(\pi_{1}(X)\right)$, pour tout $j \geq 0$. Le second résultat, dû à $\mathrm{P}$. Deligne ([D 71], voir aussi [P-S, corollary 3.6, p. 65]) affirme que tout morphisme $f: L \rightarrow M$ de structures de Hodge mixtes est strict, et en particulier, que $f(L) \cap W^{j}(M)=f\left(W^{j}(L)\right)$, pour tout entier $j \geq 0$

- Nous utiliserons la notion de morphisme strict de groupes dans la situation suivante.

Soit $h: K \rightarrow G$, et $f: G \rightarrow Q$ des morphismes de groupes de type fini tels que la composée $K \rightarrow G \rightarrow Q \rightarrow\{1\}$ soit exacte. Soit alors $s>0$ un entier, et $q_{s}^{*}: G \rightarrow G_{s}^{*}$ le quotient naturel.

On en déduit comme suit un diagramme commutatif de groupes, à lignes exactes :

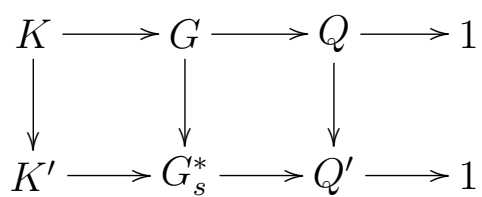

Soit $K^{\prime}:=K / K \cap \operatorname{Ker}\left(q_{s}^{*}\right)$, et $h_{s}: K \rightarrow K^{\prime}$ les quotients déduits de $h$ et $K$. Le groupe $K$ est naturellement un sous-groupe distingué (ou normal) de $G_{s}^{*}$. Soit $G_{s}^{*} \rightarrow G_{s}^{*} / K^{\prime}:=Q^{\prime}$ le quotient. Il existe alors un unique morphisme de groupes (surjectif) $Q \rightarrow Q^{\prime}$ rendant commutatif le diagramme précédent.

Les groupes $K^{\prime}$ et $Q^{\prime}$ sont donc nilpotents de classe au plus $s$, et $K^{\prime}$ est sans torsion.

Proposition 8.3 Dans la situation précédente, on suppose que $f: K \rightarrow G$ est strict. Alors :

$$
\nu\left(G_{s}^{*}\right)=\max \left\{\nu\left(K^{\prime}\right), \nu^{*}\left(Q^{\prime}\right):=\nu\left(Q^{\prime} / T\left(Q^{\prime}\right)\right)\right\}:=m
$$

Démonstration : On a, bien sûr, toujours : $\nu\left(G_{s}^{*}\right) \geq m$ sans hypothèse sur $h$. On peut supposer que $Q^{\prime}$ est sans torsion (en effet : remplacer $Q^{\prime}$ par $Q^{\prime \prime}:=Q^{\prime} / T\left(Q^{\prime}\right)$; le noyau du morphisme composé $G_{s}^{*} \rightarrow Q$ " est alors un sous-groupe $K$ " (nilpotent sans torsion) de $G_{s}^{*}$ dans lequel $K^{\prime}$ est d'indice fini. On a donc $: \nu\left(K^{\prime \prime}\right)=\nu\left(K^{\prime}\right)$. Il 
suffit alors de considérer la suite exacte $K^{\prime \prime} \rightarrow G_{s}^{*} \rightarrow Q$ " $\rightarrow\{1\}$, puisque les algèbres de Lie des complétions de Malçev de $K^{\prime}$ et $K$ " coincident, de sorte que le morphisme $K^{\prime \prime} \rightarrow G_{s}^{*}$ est également strict).

Plaçons-nous dans $L^{s}(G)=L^{s}\left(G_{s}^{*}\right)$.

(1) On a : $L^{m+1}\left(K^{\prime \prime}\right)=L\left(K^{\prime \prime}\right) \cap L^{m+1}\left(G_{s}^{*}\right)=\{0\}$, puisque $h$ est strict et $m \geq$ $\nu\left(K^{\prime \prime}\right)$.

(2) D'autre part, $L^{s} f\left(L^{m+1}\left(G_{s}^{*}\right)\right) \subset L^{m+1}\left(Q^{\prime \prime}\right)=\{0\}$, puisque $m \geq \nu(Q$ ") .

(3) Enfin, $L(K ")=K \operatorname{Ker}\left(L^{s} f\right)$, puisque $K^{\prime \prime}=\operatorname{Ker}\left(G_{s}^{*} \rightarrow Q^{\prime \prime}\right)$.

On déduit de (2) et (3) que $L^{m+1}\left(G_{s}^{*}\right) \subset L\left(K^{\prime \prime}\right)$, puis de (1) que $L^{m+1}\left(G_{s}^{*}\right)=\{0\}$.

Puisque $L^{m+1}\left(G_{s}^{*}\right)=L C^{m+1}\left(G_{s}^{*}\right)$, on a donc bien : $C^{m+1}\left(G_{s}^{*}\right)=\{1\}$

\subsection{Fibrations et classe de nilpotence des groupes de Kähler.}

Soit $X, Y$ des variétés Kählériennes compactes et connexes, et $g: X \rightarrow Y$ une fibration, c'est-à-dire : une application holomorphe surjective et à fibres connexes. Soit $X_{y}$ une fibre lisse arbitraire de $f$. L'inclusion de $X_{y}$ dans $X$ induit un morphisme de groupes $h: K:=\pi_{1}\left(X_{y}\right) \rightarrow G:=\pi_{1}(X)$, dont l'image est un sous-groupe normal de $\pi_{1}(X)$. Le morphisme de groupes $g_{*}=f: G:=\pi_{1}(X) \rightarrow Q:=\pi_{1}(Y)$ est surjectif.

La composée $g_{*} \circ h: \pi_{1}\left(X_{y}\right) \rightarrow \pi_{1}(Y)$ est triviale, mais la suite de groupes $K:=\pi_{1}\left(X_{y}\right) \rightarrow G:=\pi_{1}(X) \rightarrow Q:=\pi_{1}(Y) \rightarrow\{1\}$ n'est pas exacte en $\pi_{1}(X)$ en général (en présence de fibres multiples).

Considérons (comme en 8.3) le quotient $: q_{s}^{*}: G \rightarrow G_{s}^{*}:=\pi_{1}(X)_{s}^{*}$, puis le noyau $\mathcal{K}$ du morphisme composé $q_{s}^{*} \circ h: K \rightarrow G_{s}^{*}$, ensuite le quotient $K^{\prime}:=K / \mathcal{K}$, et enfin le quotient $G_{s}^{*} \rightarrow Q^{\prime}:=G_{s}^{*} / K^{\prime}$ (qui n'est pas a priori un quotient de $Q$, mais seulement de $G / K)$.

Théorème 8.4 Dans la situation précédente, les deux propriétés suivantes sont satisfaites :

1. $Q^{\prime}$ est un quotient de $Q$.

2. $\nu\left(G_{s}^{*}\right)=\max \left\{\nu\left(K^{\prime}\right), \nu^{*}\left(Q^{\prime}\right)\right\}$.

3. $\nu\left(G_{s}^{*}\right) \leq \max \left\{\nu\left(K_{s}^{*}\right), \nu\left(Q_{s}^{*}\right)\right\}$.

Démonstration : La propriété (3) découle de (2) et (1), puisque $K^{\prime}$ (resp. $Q^{\prime}$ ) est un quotient de $K_{s}^{*}$ (resp. $Q_{s}$ ). La propriété (2) résulte de 8.2, 8.3, et de (1). Enfin, le lemme 8.5 suivant implique aussi la propriété (1), puisque $Q^{\prime}$ est nilpotent de classe au plus $s$

Lemme 8.5 Avec les notations $K=\pi_{1}\left(X_{y}\right), G=\pi_{1}(X)$ précédentes, si $\bar{Q}:=$ $G / K$, alors pour tout entier $s>0$, le quotient naturel $\bar{Q} \rightarrow Q$ induit des isomorphismes $\bar{Q}_{s}^{*} \cong Q_{s}^{*}$. Plus généralement, tout morphisme de groupes $\bar{Q} \rightarrow H$ dont l'image est sans torsion se factorise par $Q$. 
Démonstration :Il suffit d'établir la seconde assertion, puisque les groupes $\bar{Q}_{s}^{*}$ sont linéaires sans torsion. Nous allons utiliser pour cela la base orbifolde $\Delta(g)$ de la fibration $g$ (voir [C 07]). Les assertions de l'énoncé sont invariantes par transformation biméromophe de $X$. Nous pouvons donc supposer la fibration $g: X \rightarrow Y$ nette (voir [C 07,chap. 3.2]) et telle que sa base orbifolde $(Y \mid \Delta(g))$ soit lisse (ie : $Y$ est lisse, et le support du diviseur $\Delta(g)$ est à croisements normaux).

Dans ce cas, $\bar{Q}=\pi_{1}(Y \mid \Delta(g))$ ([C 07,chap. 11.2], par exemple), et le noyau du quotient $\bar{Q} \pi_{1}(Y \mid \Delta(g)) \rightarrow Q=\pi_{1}(Y)$ est engendré par des éléments de torsion. Le lemme en résulte, puisque ces éléments ont une image triviale dans $H$

\section{Remarque 8.6}

1. Le théorème 8.4 sétend aisément au cadre des orbifoldes à multiplicités finies (grâce au lemme 8.5 ci-dessus). Par contre le cas logarithmique nécessiterait des ingrédients nouveaux (tels que ceux de [M 78]).

2. Il serait intéressant de savoir si la propriété (2) du théorème 8.4 reste vraie si l'on y remplace $G_{s}^{*}=\pi_{1}(X)_{s}^{*}$ par un quotient nilpotent sans torsion arbitraire de $G=\pi_{1}(X)$.

\section{Bibliographie}

[AN 99] D. Arapura-M. Nori. Solvable fundamental groups of algebraic varieties and Kähler manifolds. Comp. Math. 116 (1999), 173-188.

[Be 92] A. Beauville. Annulation du $H^{1}$ pour les fibrés en droites plats. LNM 1507 (1992), 1-15.

[B-S] R. Bieri-R. Strebel. Geometric invariants for discrete groups. Cours polycopié.

[Bru 99] A. Brudnyi. Classification theorem for a class of flat connections and representations of Kähler groups. Michigan J. Math. 46 (1999), 489-514.

[Ca 85] F. Campana. Réduction d'Albanese d'un morphisme Kählérien propre. I, II. Compositio Mathematica 54 (1985), 373-416.

[Ca 95] F. Campana. Remarques sur les groupes de Kähler nilpotents. Ann. Sc. ENS. 28 (1995), 307-316.

[Ca 01] F. Campana. Ensembles de Green-Lazarsfeld et quotients résolubles des groupes de Kähler. J. Alg. Geom. 10 (2001), 599-622.

[C 04] F. Campana. Orbifolds, special varieties and classification theory. Ann. Inst. Fourier 54 (2004), 499-635.

[C 07] F. Campana. Orbifoldes spéciales et classification biméromorphe des variétés Kählériennes compactes. arXiv 0705.0737.

[D 71]P. Deligne. Théorie de Hodge II. Publ. IHES 40 (1971), 5-58.

[De06] T. Delzant. L'invariant de Bieri-Neumann-Strebel des groupes fondamentaux des variétés Kählériennes. arXiv. math/0603038. (A paraître aux Math. Ann.) 
[De07] T. Delzant. Trees, valuations, and the Green-Lazarsfeld set. arXiv. math/0702477. A paraître à GAFA.

[DG 05] T. Delzant, M. Gromov. Cuts in Kähler groups. Progress in Mathematics 248. Birkhäuser 2005.

[F 83] A. Fujiki. On the structure of a complex manifold in C. Adv. Studies in Pure Math. 1 (1983), 231-302.

[GL 87] M. Green-R. Lazarsfeld. Deformation theory, generic vanishing theorems, and some conjectures of Enriques, Catanese and Beauville. Inv. Math. 90 (1987), 389-407.

[H 87] R. Hain. The De Rham homotopy of complex algebraic varieties I and II. K-theory 1 (1987), 271-324 and 481-497.

[Ka 81] O. Kharlampovich. A finitely presented solvable group with unsolvable word problem. Izv. Akad. Nauk. ser. Mat. 45 (1981), 852-873.

[M 78] J. Morgan. The algebraic topology of smooth algebraic varieties. Publ. Math. IHES 48 (1978), 137-204. Correction : Publ. Math. IHES 64 (1986), p. 185

[N] M. Namba. Branched coverings and algebraic functions. Pitman Research Notes in Mathematics Series, vol. 161. Longman Scientific and Technical (1987).

[P-S 08] C. Peters-J. Steenbrink. Mixed Hodge Structures. Erg. der Math. und ihrer Grenzgebiete. 3. Folge. Vol. 52. Springer Verlag (2008).

[R] M.Ragunathan. Discrete subgroups of Lie groups. Ergebnisse der Mathematik und Ihrer Grenzgebiete. Springer Verlag.

[Ro]D. Robinson. A course in the theory of groups. GTM 80. Springer Verlag (1996).

[Si 93] C. Simpson. Subspaces of moduli spaces of rank one local systems. Ann. Sci. ENS. 26 (1993), 361-401.

[Si 93-2] C. Simpson. Lefschetz theorem for the integral leaves of a holomorphic one-form. Compositio Math. 87 (1993), 99-113.

[U 75] K. Ueno. Classification theory of algebraic varieties and compact complex spaces. LNM 439. Springer Verlag 1975. 